\title{
ABSTRACTS OF PAPERS
}

\section{SUBMITTED FOR PRESENTATION TO THE SOCIETY}

The following papers have been submitted to the Secretary and the Associate Secretaries of the Society for presentation at meetings of the Society. They are numbered serially throughout this volume. Cross-references to them in the reports of the meetings will give the number of this volume, the number of this issue, and the serial number of the abstract.

\section{Professor B. A. Bernstein: Postulates for abelian groups} and fields in terms of non-associative operations.

The author gives postulate-sets for abelian groups in terms of "subtraction," and a postulate-set for fields in terms of "subtraction" and "division." The postulates are simple, and contain no existence proposition other than one which excludes the trivial null and one-element groups and fields. All special elements, such as "zero," "unity," are defined in terms of subtraction and division. (Received March 4, 1936.)

134. Mr. J. C. C. McKinsey: On Boolean functions of $n$ variables.

The author treats of some facts regarding the Boolean functions of $n$ variables. The results are, in part a treatment of new problems, in part a generalization to functions of $n$ variables of theorems given by Karl Schmidt (Transactions of this Society, vol. 23 (1922), pp. 212-222) for functions of one variable. First are discussed "monotone" Boolean functions (which are analogous to the monotone functions of ordinary analysis), with conditions that functions be monotone non-decreasing and monotone non-increasing. An analog is also defined and discussed, for Boolean functions, of the "continuity" of classical analysis; it is shown that all Boolean functions are "continuous" and the importance of continuity with regard to functions of Boolean variables which cannot be expressed by means of the usual Boolean operations is suggested. Finally inverse functions are discussed, and all the domains are found within which a function of $n$ variables has a one-valued inverse. (Received March 4, 1936.)

\section{Professor G. C. Evans: Sets on which a harmonic function} becomes positively infinite.

A closed set on which an otherwise harmonic function becomes positively infinite must be of capacity zero. Conversely, given a closed set of capacity zero, a function exists which becomes positively infinite at every point of the set and is otherwise harmonic. On the other hand, potentials of positive mass which become positively infinite on certain sets of category I also necessarily become infinite on enclosing sets of category II. (Received March 13,1936.) 
136. Mr. I. E. Highberg: Pseudo-polynomials in abstract spaces.

In a previous communication (abstract 41-11-418) I discussed the equivalence of two different definitions of a polynomial in a complex "espace algébrophile," hereafter called E. A. One of the definitions is as follows: A function $f(x)$ defined on one complex E. A. to another will be said to be a polynomial of the $n$th degree if (1) $f(x)$ is continuous, (2) $\Delta^{n+1} f(x) \equiv 0, \Delta^{n} f(x) \not \equiv 0$, where the increments are independent, (3) $f(x)$ is Gateaux differentiable everywhere. Under these conditions $f(x)$ can be uniquely represented in the form $f(x)=K_{0}(x)$ $+K_{1}(x)+\cdots+K_{n}(x)$, where $K_{r}(\mu x)=\mu^{r} K_{r}(x)$. In this paper I consider the form of the function when the condition (3) is suppressed. In the latter case $f(x)$ can be uniquely represented in the form $f(x)=K_{00}(x)+K_{10}(x)+K_{11}(x)$ $+\cdots+K_{n 0}(x)+\cdots+K_{n n}(x)$, where $K_{r s}(\mu x)=\mu^{r-s} \cdot \bar{\mu}^{s} K_{r s}(x)$ and $\bar{\mu}$ is the complex conjugate of $\mu$. I have ventured to call such a function a pseudo-polynomial although the terminology is somewhat conflicting. When the two complex E. A. are both the space of complex numbers, then $f(z)$ is a polynomial in the two quantities $z$ and $\bar{z}$. (Received March 12,1936.)

137. Professor A. D. Michal and Mr. E. W. Paxson: The differential in abstract linear spaces with a topology. II.

In a note presented recently to the Paris Academy, the authors defined a differential for functions on the space of the title to that same space, for which non-metric examples may be given. Some of the usual elementary properties of a differential were verified there for this "topological" differential. Here we consider further properties, in particular, giving theorems on the differentiability of iterations of differentiable functions. We also define partial differentials for functions of more than one variable. (Received March 12,1936.)

138. Dr. R. D. James: Note on formulas for the number of representations of an integer as a sum of $2 h$ squares.

There are known formulas for the number of representations of an integer $n$ as a sum of $2 h$ squares when $1 \leqq h \leqq 4$. They are of the type $\lambda \sum(-1)^{(h d-h) / 2} d^{h-1}$, where $\lambda$ may depend on the linear form of $n$ and the summation is over all $o d d$ divisors of $n$. The question arises whether formulas of this type are possible when $h \geqq 5$. In this note a simple necessary condition is developed and applied to several cases. For example, it is shown that the formula for the number of representations of $2 m, m \equiv 1(\bmod 4)$, as a sum of $2 h$ squares cannot be of the type given above if $h \geqq 7$. (Received March 13, 1936.)

139. Mr. W. A. Mersman: A new summation method for divergent series.

The method is a modification of that due to Euler-Knopp. Weighted means of the partial sums of the series are taken, the weights being binomial coefficients. However, instead of beginning with the first, we begin with the central one, the greatest. Thus the initial terms always receive the greatest weight, as in the Cèsaro-Hölder method. An expression is then found for these "partial 
sums" as a linear function of the Cèsaro means, and also as a linear function of the Euler means. Then by means of Toeplitz's theorem it is shown that (1) any series summable $(C, 2)$ is summable by this method to the same sum, and (2) any series summable $(E, 1)$ is summable by this method to the same sum. Since neither of $(C, 2)$ or $(E, 1)$ includes the other, this method is more general than either. (Received March 13, 1936.)

\section{Professor E. T. Bell: Note on an inversion formula.}

Leméray's inversion formula is shown to be a special case of Dedekind's and not, as has been asserted, a generalization. A generalization of Dedekind's formula is given. (Received March 14, 1936.)

141. Professor C. B. Morrey, Jr.: On the solutions of quasilinear elliptic partial differential equations.

In this paper, the author replaces certain linear elliptic partial differential equations of the form $a z_{x x}+2 b z_{x y}+c z_{y y}+d z_{x}+e z_{y}+f=0$ by suitable pairs of linear partial differential equations of the first order. Existence and compactness theorems for the solutions of these pairs of equations are proved under the hypothesis that the coefficients are uniformly bounded and measurable with $a c-b^{2} \geqq m>0$. These results are applied to show: (1) the existence of solutions (taking on given sufficiently smooth boundary values) of quasi-linear equations of the type $a(x, y, z, p, q) z_{x x}+2 b(x, y, z, p, q) z_{x y}+c(x, y, z, p, q) z_{y y}=d(x, y, z, p, q)$ in any case where an approximating sequence may be found with the $z_{n}, p_{n}$, and $q_{n}$ uniformly bounded; and (2) the existence and continuity of the second derivatives of any solution $z$ of $\iint_{R} f(x, y, z, p, q) d x d y=$ minimum for which the $z, p$, and $q$ are bounded. In these latter results, it is assumed only that $a, b, c$, and $d$, and the second derivatives of $f$ satisfy Hölder conditions with $a c-b^{2}>0, f_{p p} f_{q q}-f_{p q}^{2}>0$. (Received March 14, 1936.)

\section{Professor Morgan Ward: The elementary analysis of the} calculus of sequences.

In a forthcoming paper in the American Journal of Mathematics, the author has developed the formal analysis of what he terms the calculus of sequences. This calculus results on systematically replacing the factorial $1 \cdot 2 \cdots n$ by $u_{1} u_{2} \cdots u_{n}$, where $u_{1} u_{2} \cdots u_{n}$ is a fixed sequence of numbers chosen more or less arbitrarily save for the restrictions that $u_{n} \neq 0, u_{1}=1$. In this paper are defined the operations of differentiation and definite integration as applied to polynomials by limits analogous to those in the ordinary case. Among other results, Rolle's theorem and Taylor's theorem with remainder are carried over successfully. (Received March 14, 1936.)

143. Mr. D. L. Webb: Development of the algebra of n-valued logic.

Seven operations are defined in terms of a single "stroke" function in a logic of $n$ truth-values. Included among these operations are the implication and negation of Lukasiewicz and the negation and maximum of Post. The logic is symbolically complete. The matrices of these operations are determined by arithmetical means, thus allowing us to determine various properties of the 
operations and to solve the "entscheidungsproblem" for $n$ truth-values. The analogs for $n$ values of the propositions listed as most important by Whitehead and Russell in divisions 2, 3, 4, and 5 of the Principia Mathematica are tested to see which hold in the system developed. (Received March 14, 1936.)

144. Professor J. V. Uspensky: On a problem by John III Bernoulli.

If $a$ and $b$ are real numbers, $0<a<1$, and $R_{m}=[(m+1) a+b]-[m a+b]$, then the sequence $c_{0}, c_{1}, c_{2}, \cdots$ consists only of units and zeros. When $a$ is a rational number this sequence is periodic and for the formation of its period John III Bernoulli proposed remarkable rules closely related to the expansion of $a$ into a continued fraction. When $a$ is an irrational number the sequence $c_{0}, c_{1}, \cdots$ is no longer periodic. However, in any limited part of it $c_{0}, c_{1}, \cdots$, $c_{N}$ there are groups which by periodic repetition produce $c_{0}, c_{1}, \cdots, c_{N}$. Such groups may be called periods and an interesting problem arises of finding, for a given $N$, the shortest of these periods. The complete solution of this problem is given in the present paper for $b=0$ and $b=1 / 2$. In the general case the solution is more involved but no new essential difficulties arise. (Received March 16, 1936.)

145. Dr. I. M. Hostetter: Existence theorem on implicit vector functions.

This paper deals with the proof of the following theorem: Any vector equation $F\left(r_{1}, \cdots, r_{m}, \tau_{1}, \cdots, \tau_{p}\right)=0$ in the $m$ vector variables $r_{\alpha}$ and the $p$ scalar variables $\tau_{\beta}$ may be solved for any one of the vector variables $r_{i}$ in terms of the remaining variables provided that there exists a set of values $\left(r_{\alpha}\right)_{0},\left(\tau_{\beta}\right)_{0}$, which satisfy the equation; that the function $F$ is continuous and possesses continuous first derivatives in the neighborhood of this set of values; and finally, that $\left(\Delta_{r_{i}} F\right)_{n}$ does not vanish for the above set of values. The solution thus obtained is unique and continuous in the variables $r_{\alpha}, \tau_{\beta}(\alpha \neq i)$ and possesses continuous derivatives $\nabla r_{\alpha} r_{i}, \partial r_{i} / \partial \tau_{\beta}$. The function $F$ is considered as a vector in $n$-space and the vectors $r_{\alpha}$ are regarded as entities in themselves and not as functions of $n$ scalar variables. The corresponding theorem for a set of scalar equations of vector and scalar variables, or for a system of scalar equations in scalar variables, is shown to follow immediately as a consequence of this theorem. (Received March 11, 1936.)

146. Professor A. D. Michal, Mr. I. E. Highberg, and Mr. A. E. Taylor: Abstract euclidean spaces. IV.

In continuing the work on abstract euclidean spaces (see abstracts 40-11$383,41-3-158,41-9-309)$ the authors have considered rotations in various functional spaces. It has been shown that in the space of continuous functions there exists no rotation expressible as a linear functional transformation of Volterra type. In a space of functions analytic over the unit circle and continuous on the boundary, a continuous group of rotations has been obtained. The authors have also considered spaces in which the Pythagorean form $(f, g)$ is not positive definite, $(f, f) \geqq 0$. In a space of continuous functions of two variables, groups of rotations have been obtained which are not trivial. (Received March 12,1936.) 
147. Mr. A. E. Taylor: Analytic functions in general analysis. II.

The author's previously announced work on analytic functions (see abstract 41-11-424) has been continued. It is proved that if a function on one complete, complex vector space to another admits a Gateaux differential at every point of a region, and is continuous, it admits Fréchet differentials of all orders in the region. The nature of the convergence of the Cauchy-Taylor expansion is studied, with reference to compact sets, and analytic functions defined by series and integrals are considered.

Riemann's theorem on removable singularities may be generalized, but examples show that the theorems of Picard and Weierstrass concerning essential singularities fail to hold in general. A form of Mittag-Leffler's theorem is proved for abstract-valued functions of a complex variable. (Received March 12, 1936.)

148. Mr. W. A. Mersman: An existence theorem for divergent series.

Given two infinite series, each summable $(C, r)$, each term of one being greater than the corresponding term of the other, and a third infinite series each of whose terms lies between the corresponding terms of the first two series, it is proved that the third series is also summable $(C, r)$. Since the theorem is well known for $r$ equal to zero, we proceed by induction on $r$. Hardy and Littlewood (Mathematische Zeitschrift, vol. 19 (1924), p. 67) give a necessary and sufficient condition for the $(C, r)$ summability of a series in terms of the $(C, r-1)$ summability of a related series, which is precisely what is needed in order to proceed by induction with the present problem. Assuming the "related series" for the first two given series, we are able to construct the "related series" for the third series, which is then summable $(C, r-1)$ by the hypothesis of the induction, and thence by the results of Hardy and Littlewood follows the desired result. (Received March 13, 1936.)

149. Professor A. D. Michal and Mr. Victor Elconin: Differential properties of abstract transformation groups with abstract parameters. II.

In Part I of this paper (see abstract 41-11-421) certain fundamental Fréchet differential equations were obtained which are satisfied by any differentiable group function $f=f(x, \alpha)$ and its parameter function $\phi=\phi(\alpha, \beta)$, where the ranges and domains of the functions are in Banach spaces and $f(x, \alpha)$ is essential in $\alpha$.

The present Part II is devoted to the structure function $\Gamma(\alpha, \xi, \eta)$, which is defined by one of several equivalent equations, of which $\Gamma[\alpha, \Omega(\alpha, \xi), \Omega(\alpha, \eta)]$ $=d_{\eta}^{\alpha} \Omega(\alpha, \xi)-d_{\xi}^{\alpha} \Omega(\alpha, \eta)$ is an abstraction of Maurer's system. Evidently $\Gamma(\alpha, \xi, \eta)$ is bilinear and alternating in $\xi, \eta$. Moreover the complete integrability of $d_{\xi}^{\alpha} f=W(f, \Omega(\alpha, \xi))$ implies the constancy in $\alpha$ of $\Gamma(\alpha, \xi, \eta)$, and this constancy is necessary and sufficient for the complete integrability of $d_{\eta}^{\beta} \phi$ $=\Omega^{\prime}(\phi, \Omega(\beta, \eta))$, where $\Omega^{\prime}(\alpha, \xi)$ is the inverse of $\Omega(\alpha, \xi)$ with respect to $\xi$; hence, as in the classical theory, the complete integrability of the first equation implies 
that of the second. By an abstraction of Jacobi's identity, it is shown that if the first equation is completely integrable, and $W(x, \xi)$ has a continuous second Fréchet differential in $x$, then the cyclical sum $\sum_{\xi, \eta, \theta} \Gamma(\alpha, \Gamma(\alpha, \xi, \eta), \theta)$ is equal to zero. (Received March 13, 1936.)

\section{Professor A. D. Michal: General tensor analysis.}

In this paper the author continues his recent studies on general riemannian and non-riemannian differential geometries. The main portion of the paper deals with the transformation laws of general tensors and other geometric objects under Fréchet differentiable transformations of abstract Banach "coordinates." (Received March 14, 1936.)

151. Professor C. G. Latimer: On ideals in a quaternion ring and the representation of integers by Hermitian forms.

In another paper (Transactions of this Society, vol. 38 (1935), pp. 436-446) it was shown that there is a one-to-one correspondence between the classes of regular ideals in a certain quaternion ring $G$, and certain classes of binary Hermitian forms. In this paper, additional results analogous to well known results on ideals in a quadratic field, and quadratic forms are obtained. It is shown that if $m$ is prime to a certain integer determined by $G$, the number of ideals of norm $m$ is exactly $\sigma(m)$, the sum of the positive divisors of $m$. If the above mentioned forms are definite, if $f_{1}, \ldots, f_{H}$ are representatives of the classes and if $f_{i}$ has $k_{i}$ automorphs, then the total number of representations of $m$ by all the $f_{i}$ is exactly $\sigma(m)$, with the agreement that every representation by $f_{i}$ is counted as $1 / k_{i}$. A number of results in the literature on the representation of integers by quaternary quadratic forms are immediate consequences of this result. (Received March 9, 1936.)

\section{Professor Henry Blumberg: Remarks concerning mathe-} matical controversies. Preliminary report.

This paper points out and illustrates the lack of clearness and the undue domination of tradition, authority, and dogma in much of the literature of mathematical controversy; subjects to various criticisms the positions of Hilbert, Brouwer, Weyl, Russell, Gödel, and others; and suggests some common-sense ways of clarifying various important issues. (Received March 10, 1936.)

\section{Professor W. S. Kimball: New basic necessary conditions and methods in the calculus of variations.}

New conditions are established by a delta process of four stages similar to that employed in elementary calculus. In the first stage an integral is set up approximately as a sum in terms of $n$ pairs of interval variables $\Delta x, \Delta y$. In the next stage the Lagrange multiplier method for conditional extremes is applied to the "set up" before passing to the limit. The critical equations of the Lagrange method, as applied to the finite sum, become, in general, as we pass to the limit the new critical differential equations for an extremum of the integral 
limit. These critical equations exhibit new features in that (a) they are special first order equations which upon differentiation reduce to Euler's equations and include the factors $y^{\prime}$ and $1 / y^{\prime}$; (b) they combine the older method of differentials with the Lagrange multiplier method, using an isoperimetric vector condition on the differentials; (c) in physics they represent conservation of energy and momentum according to Hamilton's Principle; (d) the present method does not use or rely on the "variations" of Euler and Lagrange, nor the fundamental lemma of the calculus of variations. (Received March 10, 1936.)

\section{Dr. H. P. Doole: Integration of certain simple (step)} functions.

The integration of certain simple (step) functions, as defined by F. Riesz, has been carried out. In making a $k$-fold integration of $[x]$ it was found necessary first to develop methods of integrating various other forms of step functions. The results are applied to the evaluation of $\sum_{m=1}^{\infty} m^{1-2 k} \sin 2 \pi m x$ or $\sum_{m=1}^{\infty} m^{-2 k} \cos 2 \pi m x$ in terms of Bernoulli polynomials. The ordinary formulas are valid only in the interval $0 \leqq x \leqq 1$, but the step furiction formulas hold for any finite value of $x$. (Received March 12,1936.)

155. Dr. Ralph Hull: On quadratic rings and the arithmetics of quaternion algebras.

The author has recently shown that every maximal order $M$ of a quaternion algebra $Q=(a, Z)$ is of the form $\mathfrak{M}=\mathfrak{m}_{c}+(\lambda+u) \mathfrak{n}$, where $\mathfrak{m}_{c}$ is an order (quadratic ring) in $Z$ of conductor $c$ prime to the reduced discriminant of $Q, \mathfrak{n}$ is an $\mathfrak{m}_{c}$-modul and $\lambda$ is in $Z$. (See abstract 42-1-1.) In this paper, the genera of regular $\mathfrak{m}_{c}$-ideals are studied and the results are applied to the converse problem of determining all $\mathfrak{n}$ and $\lambda$ for a given $\mathfrak{m}_{c}$ such that $\mathfrak{m}_{c}+(\lambda+u) \mathfrak{n}$ is an $\mathfrak{M}$, and to the determination of the $\mathfrak{M}$-ideals of $Q$ for an arbitrary $\mathfrak{M}$. (Received March $13,1936$.

\section{Professor G. A. Bliss: Definitely self-adjoint boundary- value problems.}

In an earlier paper (Transactions of this Society, vol. 28 (1926), pp. 561-584) the author gave a definition of boundary value problems which are self-adjoint or definitely self-adjoint. The boundary value problems associated in a well known way with non-singular problems of the calculus of variations are all self-adjoint, and those problems which arise out of non-singular problems of the calculus of variations without differential equations as side conditions are definitely self-adjoint. In the present paper, a modification of the definition of definite self-adjointness is given which is applicable also to boundary value problems arising from non-singular problems of the calculus of variations of the very general type formulated by Bolza. Such a boundary value problem is definitely self-adjoint provided that the problem of Bolza has the property of normality. For the newly defined definitely self-adjoint boundary value problems proofs are given of the reality of the characteristic numbers, the equality 
of the index and multiplicity of a characteristic number, and the expansion theorems. (Received March 13, 1936.)

157. Dr. W. T. Reid: A system of ordinary linear differential equations with two-point boundary conditions.

This paper treats the boundary value problem consisting of the differential equations $y_{i}^{\prime}=\left[A_{i j}(x)+\lambda B_{i j}(x)\right] y_{j},(i, j=1, \cdots, n ; a \leqq x \leqq b)$, and $n$ linearly independent boundary conditions $s_{i}[y] \equiv M_{i j} y_{j}(a)+N_{i j} y_{j}(b)=0$. This system is supposed to satisfy the following hypotheses: (1) it is self-adjoint under a transformation $z_{i}=T_{i j}(x) y_{j}$; (2) $\left\|S_{i j}(x)\right\| \equiv\left\|T_{k i}(x) B_{k j}(x)\right\|$ is symmetric; (3) $u_{i} S_{i j}(x) u_{j} \geqq 0$ for $\left(u_{i}\right)$ real; (4) there is no non-identically vanishing solution of $y_{i}^{\prime}=A_{i j}(x) y_{j}, s_{i}[y]=0$ such that $B_{i j} y_{j} \equiv 0$ on $a b ;(5)\left\|B_{i j}(x)\right\|$ has constant rank $n-r(0 \leqq r<n)$ on $a b$. The above hypotheses (1)-(4) are the characteristic properties of a definitely self-adjoint boundary value problem according to a modified definition of definite self-adjointness as recently proposed by Bliss. (See abstract 42-5-156.) Under the hypotheses listed above this problem is here shown to be equivalent in a new way to a boundary value problem associated with the second variation of a calculus of variations problem, and of the type previously considered by the author (American Journal of Mathematics, vol. 54 (1932), pp. 769-790). Results concerning the existence of characteristic values, expansion theorems, and certain comparison and oscillation theorems for the system here treated are then consequences of corresponding theorems for the system previously considered. (Received March 13, 1936.)

158. Professor Dunham Jackson: The diagonal summation of double Fourier series.

Diagonal summation of the Fourier series for a function of two real variables has been discussed by Küstermann in his dissertation (Munich, 1913). It appears that the relevant properties of the function are similar to those in the case of ordinary summation by squares, except that the special significance of lines parallel to the coordinate axes in the $(x, y)$-plane is transferred to lines inclined at $45^{\circ}$ to the axes. The present paper points out that this correspondence is a priori necessary, inasmuch as the diagonal partial sums in terms of $x$ and $y$ are identical with the square partial sums of the Fourier development of the same function in terms of the variables $\xi=(x+y) / 2, \eta=(x-y) / 2$. The direct treatment of diagonal summation is nevertheless of some interest, and is further studied for its own sake. (Received March 13, 1936.)

159. Mr. A. E. May: On the rational equivalence of pairs of Hermitian matrices.

Consider two pairs of $2 \times 2$ Hermitian matrices, $P_{1}, P_{2}$, and $Q_{1}, Q_{2}$, with elements in $R(\sqrt{ } k)$, where $k$ is a negative integer containing no integral square other than 1 . Known necessary conditions for the equivalence of these pairs in $R(\sqrt{ } k)$ are that $\left(P_{2}-\lambda P_{1}\right)$ and $\left(Q_{2}-\lambda Q_{1}\right)$ have the same invariant factors and that $P_{1}\left(P_{1}^{-1} P_{2}-\lambda I\right)^{n}$ and $Q_{1}\left(Q_{1}^{-1} Q_{2}-\lambda I\right)^{n}$ have the same index for all real $\lambda$ and all positive integral $n$. Let $P_{1}$ and $Q_{1}$ be equivalent in $R(\sqrt{ } k)$ and non-singular. Assuming that $P_{1}, P_{2}$, and $Q_{1}, Q_{2}$ satisfy the above conditions, a canonical form 
is developed which determines a certain finite system of integral binary quadratic forms. The original pairs will be equivalent if and only if one of the forms of the system represents the norm of an integer in $R(\sqrt{ } k)$. (Received March 13, 1936.)

160. Professor R. L. Jeffery: Functions of bounded variation in two or more variables.

In recent papers (Transactions of this Society, vol. 35 (1933), pp. 824-854; vol. 36 (1934), pp. 1-30) C. R. Adams and J. A. Clarkson have studied the relations between the various definitions for functions of two real variables. It appears from their investigations that none of the definitions so far given is a direct extension of the definition of bounded variation for a function of a single real variable. If $F(x)$ is of bounded variation there exists a sequence of summable functions $s_{n}(x)$ with $\int_{e} s_{n} d x$ bounded in $n$ and $e$, for which $F(x)$ $=\lim \int_{a}^{x} s_{n} d x$. This property of $F(x)$ can be extended to functions of two variables: $F(x, y)=\lim \int_{0}^{x} \int_{0}^{x} s_{n}(x, y) d x d y, \int_{e} s_{n} d x d y$ bounded in $n$ and $e . F(x, y)$ is bounded; its discontinuities form a set of zero measure; it can be expressed as the difference of two monotone functions. Associated with $F(x, y)$ is an additive function of intervals $F(\omega)=\lim \int_{\omega} s_{n} d x d y . F(\omega)$ is of bounded variation. If $F^{\prime}(x, y)=\lim _{m \omega \rightarrow 0} F(\omega) / \omega, \omega$ a square with center $(x, y), F^{\prime}$ exists and is finite almost everywhere and is summable. The functions $F$ and $F^{\prime}$ may exist when $\int_{e} s_{n} d x d y$ is not bounded in $n$ and $e$. If $F^{\prime}$ exists and is finite then $F$ can be determined in at most a countable set of operations. (Received March 14, 1936.)

\section{Dr. Max Zorn: Generalization of the associative law.}

A complete description of a certain type of associative laws for linear algebras is given in this paper. (Received March 14, 1936.)

162. Dr. Max Zorn: On the second fundamental theorem in the theory of alternative algebras.

Two proofs are given of the fact that an alternative division algebra of finite rank is a Dickson generalization of Cayley's non-associative algebra with eight units. The first has already been sketched by the author in a previous paper; the second avoids as much as possible the theory of idempotents in alternative algebras. (Received March 14, 1936.)

163. Dr. E. F. Beckenbach: $S u b-F(x ; \alpha, \beta)$ functions.

Let $F(x ; \alpha, \beta)$ be a two-parameter family of real continuous functions defined in an interval $(a, b)$, such that there is a unique member of the family taking on arbitrary values $y_{1}, y_{2}$ at arbitrary points $x_{1}, x_{2}$ of the interval. A real function $f(x)$ will be said to be a sub- $F(x ; \alpha, \beta)$ function in $(a, b)$ provided, for arbitrary $x_{1}, x_{2}$ in $(a, b), f(x) \leqq F_{12}(x), x_{1} \leqq x \leqq x_{2}$, where $F_{12}(x)$ is the $F(x ; \alpha, \beta)$ satisfying $F_{12}\left(x_{1}\right)=f\left(x_{1}\right), F_{12}\left(x_{2}\right)=f\left(x_{2}\right)$. For instance, for a convex function, $F(x ; \alpha, \beta)=\alpha x+\beta$, and for the Phragmén-Lindelöf function $h(\theta)$ of complex function theory, $F(\theta ; \alpha, \beta)=\alpha \cos \rho \theta+\beta \sin \rho \theta$. The properties of and necessary and sufficient conditions for sub- $\mathrm{F}(x ; \alpha, \beta)$ functions are discussed. (Received March 14, 1936.) 
164. Professor A. T. Craig: A certain mean value problem in statistics.

In this paper the arithmetic mean value of the sum of the squares of $n$ normally and independently distributed variables is found by analytic methods when those variables are subject to $m<n$ linear restrictions. (Received March 14, 1936.)

165. Dr. E. G. Harrell: On the topology of a two-parameter, non-metric, and non-separable space.

In this paper is given a discussion of a special space $S$ which is non-metric and non-separable, but which admits extensions of topological properties of the euclidean space. $S$ is a space which is the product of a simply ordered continuous series $K=K[x]$ by itself. The points $x$ of $K$ are symmetrical or nonsymmetrical with respect to the order types of the points $x^{\prime}<x$, and $x^{\prime \prime}>x$ and the topological properties of the subcontinua of $K$ and likewise of $S$ are studied with respect to this factor. Detailed proofs of theorems on polygons which are true on the basis of the existence of rectangles in $S$ are given, and the Jordan theorem is then demonstrated for this space by simple extension of proofs by Kerékjártó and Diénes. (Received March 14, 1936.)

166. Professor G. Y. Rainich: Remark on the deviation from analyticity of a non-analytic function.

A given non-analytic function possessing a differential at a point is compared with an arbitrary analytic function in the neighborhood of that point. The limit of the maximum of the absolute value of the difference over a circular disk, divided by the radius is defined as the deviation from the analytic function considered. The minimum of this deviation is found to be the absolute value of the areal derivative of the given non-analytic function; it is attained for a whole class of analytic functions. (Received March 14, 1936.)

\section{Mr. R. W. Wagner: Canonical form as a many-valued} function in matrix space. Preliminary report.

As a preparation for studying many-valued analytic functions of a matrix, the characteristic roots and canonical form, in terms of which the other functions are usually defined, should be considered as functions of the matrix. Square matrices of the $n$th order with complex elements are considered as points of a "matrix space" of $2 n^{2}$ dimensions. This space has a complicated structure, and one essential feature is the existence of a sub-locus $S$ of matrices with equal roots. The study of many-valuedness of the canonical form involves the topological properties of the complement of $S$. For the case $n=2$, it is shown that $S$ consists of two intersecting branches and that if the matrix is taken around a path which links one, and only one, branch of $S$, the two associated canonical forms are interchanged instead of returning to their original values. A method is indicated for constructing a "Riemann" matrix space in which the canonical form function is single-valued. (Received March 14, 1936.) 
168. Mr. Herbert Boggs and Professor G. Y. Rainich : Note on group postulates.

In this paper a set of four postulates is given involving two operations which are connected by one of the postulates (Postulate 3). Multiplication is defined in terms of these two operations, which are called left and right divisions; the associative law of multiplication is translated into a form involving the two divisions only. It is then shown that the set of postulates defines a group by deriving the closure property and the other properties in the definition of a group. The set of four postulates is equivalent to a set of three using multiplication offered by Garver in this Bulletin, vol. 40 (1934), pp. 698-701. The position that the set of four postulates, using two divisions, offers a keener analysis of the situation, is justified by proving the independence of postulate 3 ; that is, a realization is exhibited where there does not exist a connection between the two divisions and where the other three postulates are true. (Received March 14, 1936.)

169. Mr. L. E. Mehlenbacher: The interrelations of the fundamental solutions of the hypergeometric equation.

The object of this paper is to extend and complete the earlier researches of Lindelöf, Barnes, and others relative to the connecting constants which join the fundamental solutions of the hypergeometric equation about its singular point $z=0$ with those about its singular points $z=1$ and $z=\infty$. The earlier researches have been chiefly confined to those cases in which the difference of the exponents at each singular point is not an integer, but the results are now extended to other cases; namely, cases in which logarithmic solutions of the differential equation present themselves. An exhaustive study is made of all possible situations and the corresponding interrelations of the solutions. (Received March 16, 1936.)

170. Professor C. C. MacDuffee: On a fundamental theorem in matric theory.

This paper contains a short proof of the well known theorem on the relation of the elementary divisors of the matrix $\phi(A)$ to the elementary divisors of $A$, where $A$ is any matrix and $\phi$ any polynomial in an algebraically closed field $F^{*}$. A short proof is also given of McCoy's extension of this result to a general field $F$. (Received March 16, 1936.)

171. Professor Fritz John: Identities between certain sums and integrals.

An earlier paper by the author (Mathematische Annalen, vol. 110 (1934), pp. 718-721) dealt with certain sums involving an arbitrary function, which could be expressed as integrals. These results were extended by $\mathrm{H}$. Rademacher (American Journal of Mathematics, vol. 58 (1936), pp. 169-176). The present paper gives other generalizations. Let $f(x)$ for $x>0$ be a continuous function of bounded variation satisfying a functional equation $f(p x)=p f(x)$ with some $p>1$. Let $F(x, z)=\sum_{\mu=1}^{\infty}(-1)^{\mu+1} f[x /(\mu-z)]$. Then $F(x, z)=\int_{1}^{p} f(x / y) d \psi_{p}(y, z)$ for $0<z<1$, where $\psi_{p}(y, z)=\sum_{\alpha=-\infty}^{\infty}\left[\left(2 y p^{\alpha}+2 z\right)-2\left(y p^{\alpha}+z\right)\right] / p^{\alpha}$. If in par- 
ticular $f$ has a continuous derivative and $p$ is an odd integer greater than 1 , then also $\int_{-z}^{1-z} F(x,-y) d \psi_{p}(y, z)=\sum_{\mu=1}^{\infty}(-1)^{\mu}\left\{\log _{p}(\mu-z)(1-z)^{-1}\right\} f[x /(\mu-z)]$, where $\{x\}$ denotes the smallest integer $\geqq x$. The integrals are RiemannStieltjes integrals. (Received March 16, 1936.)

172. Dr. O. K. Sagen: The integers represented by sets of binary biquadratic forms. Preliminary report.

A set $\Sigma(I, J)$ of positive binary biquadratic forms having discriminant $D=I^{3}-27 J^{2}$ is defined as the set of all forms having the same invariants $I, J$. A given integer is represented by the set $\Sigma(I, J)$, if there exists a form in the set which represents the integer. It is shown that under certain conditions a set $\Sigma(I, J)$ is regular, that is, all integers not represented by the set lie in arithmetic progressions, while in general sets $\Sigma(I, J)$ are not regular. (Received March 16, 1936.)

173. Professor Cornelius Lanczos: $A$ simple recursion method for solving a set of linear equations.

In the problem of solving a set of simultaneous linear equations with nonvanishing determinant both the application of determinants and the customary elimination method involve laborious calculations. Based on the principle of successive orthogonalization of a set of vectors a recursion method has been found which replaces the original set of equations by a new set in which, however, all the coefficients to the right of the diagonal are missing so that each successive step involves but one new unknown. Hence, the new set of equations can be solved numerically by proceeding successively from one equation to the next. The coefficients of the auxiliary set are themselves determined by successive recursions. (Received March 19, 1936.)

174. Professor C. R. Adams: The space (BV) of functions of bounded variation.

For this space the "classical" metric is $(x, y)=|x(0)-y(0)|+T_{0}{ }^{1}(x-y)$, where $T_{0}{ }^{1}(z)$ represents the total variation of $z(t)$ on $0 \leqq t \leqq 1$. Although natural and suitable for the subspace $(A C)$ of absolutely continuous functions, this metric seems from some standpoints undesirably strong for $(B V)$, which thus metrized is not separable. Recent papers by Clarkson, Lewy, and the present writer on convergence in variation (this Bulletin, vol. 40 (1934), pp. 413-417) and on convergence in length (Duke Mathematical Journal, vol. 1 (1935), pp. 19-26) suggest several possible new metrics for $(B V)$. Of these $(x, y)$ $=\int_{0}^{1}|x(t)-y(t)| d t+\left|T_{0}{ }^{1}(x)-T_{0}{ }^{1}(y)\right|$ is perhaps the most suitable; it is generally employed in this paper. So metrized, $(B V)$ is complete and separable, and every bounded portion of the space is compact; since in general $(x, y)$ $\neq(x-y, \theta)$ and $\lim \left(x_{n}+y_{n}\right) \neq \lim x_{n}+\lim y_{n}$, it is not a normed vector space. This metric imposes a distinction between vector equality and metric equality. Continuity of an additive operation at one point of $(B V)$ does not imply continuity everywhere, and an operation on $(B V)$ may be additive and continuous without being uniformly continuous. The general form of an additive and uniformly continuous functional on $(B V)$ is $\int_{0}^{1} x(t) \alpha(t) d t$, where $\alpha(t)$ is summable and essentially bounded. (Received March 2, 1936.) 
175. Professor R. P. Agnew: Products of methods of summability.

This note gives a simple example of two matrices $\left\|a_{n k}\right\|$ and $\left\|b_{n k}\right\|$ defining regular methods of summability $A$ and $B$ and a sequence $\left\{s_{n}\right\}$ which is summable $B$ to $O$ while $\left\{s_{n}\right\}$ is non-summable by the method $A B$ defined by the matrix $\left\|c_{n k}\right\| \equiv\left\|a_{n k}\right\|\left\|b_{n k}\right\|$. It follows that regularity of $A$ and $B$ does not imply $A B \supset B$. The transformation $A$ is equivalent to $I$ (the identity) and it follows that regularity of $A B$ and $D$ and equivalence of $A$ and $D$ do not imply $A B \supset D B$. Applications to kernel transformations are given. (Received March 3, 1936.)

176. Professor W. D. Cairns: A theorem in the theory of numbers as applied to regular polygons.

If the circumference of a unit circle be divided into $n$ equal parts, a line drawn from one point of division to the $k$ th point of division, counting therefrom, ( $k$ prime to $n$ ) will be the side of a regular polygon of $n$ sides. At the St. Louis meeting of the Mathematical Association of America the author showed by three different methods that the sides of the $\phi(n) / 2$ possible $n$-gons are the roots of an equation of the $n$th degree with simple coefficients. The general theorem depends on an existence theorem which is proved in the present paper. (Received March 13, 1936.)

177. Professor Leonard Carlitz: On certain equations in a relative-cyclic field.

Let $k$ be an arbitrary commutative field (of characteristic 0 or $p$ ), and $K / k$ relative-cyclic of degree $m$. If the Galois group of $K / k$ be generated by $S$, and $\alpha$ and $\beta$ be elements of $K$, then the equations in question are: (i) $\xi^{S}=\alpha \xi$, and (ii) $\xi^{S}=\alpha \xi+\beta$. If $K$ be defined by $f(t)$, an irreducible polynomial in $k[t]$, we set up the isomorphism $K / k \cong k[t] / f(t)$; let $a(t), b(t)$ correspond to $\alpha, \beta$, respectively. Then (i) has a solution $\neq 0$, if and only if $R(a, f)$, the resultant of $a(t)$ and $f(t),=1$. As for (ii), in the case $\alpha \neq \gamma^{S-1}$, the equation has a unique solution $\xi$ for each $\beta$; in the case $\alpha=\gamma^{S-1}$, we may put $\alpha=1$, and then the condition for solvability is that the coefficient of $t^{m-1}$ in $a(t) f^{\prime}(t)$, reduced $\bmod f(t)$, be zero. (Received March 13, 1936.)

\section{Mr. J. J. De Cicco: Geometry of whirl series.}

The following theorems on series of lineal elements are generalizations of certain theorems developed by Professor Kasner. Theorem I: Under any transformation there exists an $\infty^{4}$ set of curves which are carried into whirl series. Any transformation which carries every curve into a whirl series must be the product of a contact transformation by a whirl. Theorem II: Under any transformation there exists an $\infty^{5}$ set of equitangential series which are carried into whirl series. Any contact transformation which carries every equitangential series into a whirl series must carry every equitangential series into an equitangential series. Any transformation which carries every equitangential series into a whirl series must be the product of an equi-long transformation by an expansion by a whirl. Theorem III: Under any transformation there exists an $\infty^{5}$ set of isogonal series which are 
carried into whirl series. Any contact transformation which carries every isogonal series into a whirl series must carry every isogonal series into an isogonal series. Any transformation which carries every isogonal series into a whirl series must be the product of a conformal transformation by a whirl. Theorem IV: Under any transformation there exists an $\infty^{7}$ set of whirl series which are carried into whirl series. Any element transformation which carries every whirl series into a whirl series must be the product of a rigid motion by an expansion by a whirl. (Received March 11, 1936.)

179. Mr. Nelson Dunford and Mr. Anthony Morse: Remarks on a preceding paper of $J$. A. Clarkson.

Let $X$ be a Banach space having a base $\left\{x_{i}\right\}$ such that $\sum_{i=1}^{\infty} a_{i} x_{i}$ converges whenever $\left\|\sum_{i=1}^{\infty} a_{i} x_{i}\right\|$ is bounded. It is shown that an additive function on elementary figures in euclidean $n$-space to $X$ which is of bounded variation has a summable derivative; an absolutely continuous function is the indefinite integral of its derivative. The theorem applies to Hilbert space, $l_{p}(p \geqq 1)$ and $L_{p}(p>1)$. (Received March 13, 1936.)

180. Professor L. A. Dye: An involutorial transformation determined by three pencils of planes.

Two projective pencils of planes $|u|$ and $|v|$ are each put into a $(1,2)$ correspondence with a third pencil of planes $|p|$ which is in involution. The intersections of corresponding planes of $|u|$ and $|v|$ are the generators $r$ of a quadric surface $H$. A point $P$ of space determines a plane $p$, and hence its conjugate $\bar{p}$, a pair of planes $u, v$, and their line of intersection $r$. The plane determined by $(P, r)$ is tangent to $H$ at a point $Q$. The line $P Q$ meets $\bar{p}$ in a point $P^{\prime}$, the image of $P$ in an involutorial transformation of order 7 . The locus of the piercing points of the lines $r$ and the corresponding planes $p, \bar{p}$ is a curve of order 5 along which the homoloidal surfaces $S_{7}$ touch each other. The axis $l$ of $|p|$ is a five-fold fundamental line along which the tangent planes to any $S_{7}$ are fixed at every point for all five sheets. The transformation is generalized and a transformation of order $4 n+3$ is discussed. (Received March 11, 1936.)

181. Professor H. T. Engstrom and Dr. Max Zorn: Transformations of reference systems in the space-time of Page.

In his paper, $A$ new relativity, Leigh Page has constructed reference systems which (1) allow a euclidean geometry and a time definition such that the light travels with a constant speed $c$ along straight lines and (2) are not Lorentz transforms of an underlying inertial system. He proposed the problem of determining all the reference systems with the property (1). The present paper answers this question by showing that Page's problem is equivalent to the determination of the coordinate transformations which leave the differential equation $I=d x^{2}+d y^{2}+d z^{2}-c^{2} d t^{2}=0$ invariant. One obtains a 15 -parameter continuous group, the conformal group belonging to the differential form $I$. This is done in the Lie theory of continuous groups which also shows that the preservation of $I=0$ already implies (1) straightness of light paths and (2) the invariance of the quotient of physical intervals. Page's theory may be consid- 
ered as the special relativity belonging to the conformal general relativity of Weyl. (Received March 9, 1936.)

\section{Mr. Aaron Fialkow: Initial motion of fields of force.}

In previous papers $\mathrm{E}$. Kasner and the writer obtained theorems concerning the geometry of the initial motion of a particle starting from rest in a positional field of force. These results were stated in terms of the ratio set which Kasner showed to be 3 in general. The ratio set is defined as the set of limiting ratios of the infinitesimal departures of the initial line of force and of the path from their common tangent. The author now shows that the ratio set is also determined by the isocline through the initial point, and also examines the geometry of the locus of points where the ratio set is not 3. (Received March 4, 1936.)

\section{Professor Philip Franklin: A new class of transcendental} numbers.

Hilbert's conjecture that an irrational algebraic power of any algebraic number except zero and one is transcendental has recently been established by Geifond and, independently, by Schneider. G. Ricci has shown that the transcendentality persists if the algebraic numbers are replaced by products of algebraic numbers and a restricted type of Liouville number. The author introduces a class of transcendental numbers, $A$, related to a type discussed by Ore, bearing a similar relation to the numbers of an algebraic field that the Liouville numbers do to the rationals. His principal theorem asserts the transcendentality of a power, whose base and exponent are both restricted types of numbers $A$. The results include those of Ricci, and the Gelfond-Schneider result, as special cases. A result on quotients of logarithms specializes to a theorem like those of Ricci, but not treated by him. Since a combination of ideas due to Gelfond and Schneider has been used, the method gives a slightly simplified proof of their original theorem. The totality of numbers shown to be transcendental by these theorems has zero measure. (Received March 11, 1936.)

\section{Professor J. J. Gergen: Summability of double Fourier} series.

Let $f(u, v)$ be integrable $L$ over $(0,0 ; \pi, \pi)$, even and periodic with period $2 \pi$ in each variable. Let $(A)$ be the Fourier series of $f$ at the origin. In a recent paper (Transactions of this Society, vol. 38 (1935), pp. 401-435) Dr. Littauer and the author obtained the following result. If $0 \leqq \alpha<a-2,0 \leqq \beta<b-2$, if $(A)$ is summable $(C ; \alpha, \beta)$ to sum $s$, and if $f$ is bounded $(C ; a, b)$ in some square $(+0,+0 ; \delta, \delta), 0<\delta$, then $f$ is continuous $(C ; a, b)$ at the origin with limit $s$. In the present paper the corresponding result obtained by interchanging the roles of continuity and summability is considered. For each pair $0<\alpha, 0<\beta$ an $f$ is defined which vanishes for $0 \leqq u \leqq \pi / 2,0 \leqq v \leqq \pi$, for which $(A)$ is bounded $(C ; \alpha, \beta)$, but for which $(A)$ is not summable $(C ; \alpha, \beta)$. It is shown, however, that, if $0 \leqq \alpha<a-1<\xi-1,0 \leqq \beta<b-1<\eta-1$, if $f$ is continuous $(C ; a, b)$ at the origin with limit $s$, if $(A)$ is ultimately bounded both $(C ; \alpha, \eta)$ and $(C ; \xi, \beta)$, 
then $(A)$ is summable $(C ; \xi, \eta)$ to sum $s$. Analogous results hold for Riesz summability. (Received March 14, 1936.)

185. Professor L. M. Graves: On the completing of a Hausdorff space.

Quite recently Garrett Birkhoff has indicated how the notion of MooreSmith convergence may be used to give a method for completing a linear Hausdorff space which does not satisfy the first countability axiom. In this note it is shown that the method may still be used in a regular Hausdorff space which need not be linear, but into which a uniformity is introduced through a certain correspondence between the neighborhoods of different points. (Received March 18, 1936.)

186. Dr. M. R. Hestenes: Minimax principle in the calculus of variations.

In the present paper a modification is given of the generalized minimax principle of Birkhoff and Hestenes (Duke Mathematical Journal, vol. 1 (1935), pp. 413-432; cf. Morse, American Mathematical Society Colloquium Publications, vol. 18). A preliminary report of a portion of this paper is given in the Proceedings of the National Academy of Sciences, vol. 22 (1936). In particular there is given a topological definition of critical sets of type $k$ for an arbitrary function on a metric space. This definition is based on an extension of the notion of minimizing sequences used in the absolute minima theory. In this sense the author's method is closely allied to the methods of Hilbert, Tonelli, McShane, Graves, and others. The results are applied to simple integral problems in the calculus of variations. It is found that critical sets of type $k$ always exist for positive definite positively quasi-regular integral $J$ on a complete class $K$ of rectifiable arcs whose $k$ th connectivity is non-null. If $J$ is also positively regular, every curve of a critical set is in general an extremal arc. No special treatment is needed for closed extremals. (Received March 13, 1936.)

\section{Professor T. R. Hollcroft: Relations among characteristics of associated linear systems.}

In his treatment of the web of plane curves, Caporali (Sopra i sistemi lineari triplamente infiniti di curve algebriche piane, Collectanea Mathematica in Memoriam Dominici Chelini (1881), pp. 144-170) discusses fully the loci of singular points of curves of the web, but, although he derives the properties of contact loci, he does not define or discuss them as such. An inquiry into the reasons for this omission together with the study of the corresponding problem in linear systems of surfaces and manifolds leads to the conclusion that the relations of contact loci in linear systems are not at all analogous to those of singularity loci. The two following statements are established: (1) In any linear system $R$ of algebraic manifolds, the singularity loci of $R$ are generating elements, respectively, of the singularity loci of any linear system of higher grade (Stufe) containing $R$. (2) In any linear system $R$ of algebraic manifolds, the contact loci of $R$ are not generating elements of the contact loci of any linear system of higher grade containing $R$. (Received March 20,1936.) 
188. Dr. Charles Hopkins: Concerning groups of order $p^{m}$ whose operations are of order $\leqq p$. Preliminary report.

If the elements of a group $G$, of order $p^{m}$, are all of order $p$, the identity $E$ excepted, and if $H$, of order $p^{r}$, is a maximal invariant abelian subgroup of $G$, then $G / H$ is a subgroup of the group of isomorphisms of $H$ (Brahana). One can show that $G$ is a subgroup of the $\operatorname{SLH}(r+1 ; p)$; that the elements of $G$ may be represented in the form $E+x_{1} u_{1}+\cdots+x_{m} u_{m}$, where the $x$ 's are elements of the $G F(p)$, the $u$ 's are a normal basis of a nilpotent algebra $N$, and $E$ is a principal unit of the linear associative algebra $A=\{E, N\}$ over the $G F(p)$. The object of this investigation is to exhibit relationships between the ideal structure of $A$ and the commutator structure of $G$. (Received March 14,1936.)

189. Mr. I. N. Kagno: The triangulation of surfaces and the Heawood color formula.

In this paper are proven a number of theorems on the triangulation of surfaces by graphs. It is shown that if a graph $G$ can be mapped on a surface $\subseteq$

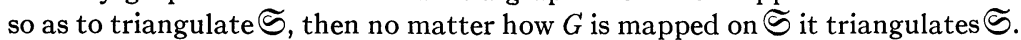
The author applies the special case where $G$ is a complete $N$-point to the map coloring problem. P. J. Heawood (Quarterly Journal of Mathematics, vol. 24 $(1890)$, p. 332) gave a formula for $\left[P_{K}\right]$, (where $[x]$ denotes the greatest integer in $x$ ) an upper bound to the number of mutually touching regions which can be mapped on a surface of characteristic $K$. This number is also an upper bound to values of $N=P_{K}$, such that a complete $N$-point can be mapped on the same surface. It is shown that when $K$ is even and $\left[P_{K}\right]=P_{K}$, a complete $N$-point cannot be mapped on one of the two surfaces: (a) the orientable surface of characteristic $K$, (b) the non-orientable surface of characteristic $K$. For such exceptions, the Heawood formula then gives a necessary, but not sufficient condition. (Received March 2, 1936.)

190. Dr. E. R. van Kampen: A unicity theorem for ordinary differential equations and a postulate system for Lie groups.

If a system of equations $d y^{\nu} / d t=C^{\nu}(y), C^{\nu}(y)$ continuous, has a solution of the form $y^{\nu}=f^{\nu}(x, t), f^{\nu}(x, 0)=x^{\nu}$ satisfying a uniform Lipschitz condition in the initial values $x^{\nu}$ and forming the equations of a 1-parameter Lie group, then the system has no other solutions. This theorem combined with an elementary theory of the foundation of Lie groups (worked out several years ago, but never published) leads to the following statement: if a topological group nucleus, homeomorphic with an $n$-cell, has a coordinate system in which the product of two elements has continuous derivatives to the coordinates of the first element and satisfies a Lipschitz condition in the coordinates of the second element, then the group nucleus is a Lie group nucleus. (See abstract 41-5-192.) (Received March 13, 1936.)

\section{Professor Edward Kasner: General harmonic transforma- tions.}

The author studies transformations $X+i Y=\phi(x, y)+i \psi(x, y)$ where $\phi$ and $\psi$ are arbitrary harmonic functions; so that the Laplace equations are satisfied but not necessarily the Cauchy-Riemann equations. A characteristic property 
from the viewpoint of polygenic functions is that the mean derivative of the given polygenic function is then monogenic (see Science, vol. 66 (1927), p. 581). The class of all harmonic transformations does not form a group but, of course, includes the set of direct and reverse conformal transformations as a subgroup. Particular cases of harmonic polygenic functions $w=f(z)+g(\bar{z})$ have been studied by Schwarz (1887) in connection with minimal surfaces. He also discusses the condition for singular points. In the present paper several general properties are obtained. Finally the theory is extended to three and $n$ dimensions, though here, of course, one cannot use functions of a complex variable. (Received March 12, 1936.)

\section{Dr. H. L. Krall: On derivatives of orthogonal polynomials.}

Let $\left\{\phi_{n}(x)\right\}$ be a set of polynomials, orthogonal in a finite interval $(a, b)$ with the weight function $p(x)$. It has been shown by W. Hahn (Mathematische Zeitschrift, vol. 39 (1935)) that if the derivatives $\left\{\phi_{n}^{\prime}(x)\right\}$ also form an orthogonal set, then the $\left\{\phi_{n}(x)\right\}$ are Jacobi polynomials. A new proof of this result is given by showing that if $\left\{\phi_{n}(x)\right\}$ and $\left\{\phi_{n}^{\prime}(x)\right\}$ are orthogonal sets, the function $p(x)$ is the weight function of the Jacobi polynomials. (Received March 21, 1936.)

\section{Dr. H. L. Krall: On higher derivatives of orthogonal poly-} nomials.

By a method similar to that of the preceding paper, it is shown that, if $\left\{\phi_{n}(x)\right\}$ is a set of polynomials orthogonal in a finite interval $(a, b)$, and if the $r$ th derivatives $\left\{\phi_{n}{ }^{r}(x)\right\}$ form a set of polynomials orthogonal in an interval $(c, d)$ (finite or infinite), then the original set $\left\{\phi_{n}(x)\right\}$ are Jacobi polynomials. (Received March 21, 1936.)

194. Professor R. E. Langer: On the determination of earth conductivity from observed surface potentials.

In previous papers L. B. Slichter and the author have considered the problem of calculating subterranean electrical conductivities from the data supplied by measurements of the surface potentials present about an electrode which is supplying current to the earth. The solution obtained was in the form of a power series for the conductivity as a function of the depth. Such a solution, from its nature, is not adapted to a determination of discontinuities in the conductivity, such as might result from stratification of the earth. The present paper extends the analysis of the surface potentials, and shows how the location and magnitude of the first discontinuity of the conductivity, or of its derivative, can be computed. (Received March 19, 1936.)

195. Dr. Saunders MacLane: A combinatorial condition for planar graphs.

Let a graph in a plane, consisting of a number of Jordan arcs joined at certain points (or vertices), subdivide the plane into regions. Each region is bounded by a circuit, and these circuits together form a fundamental system 
of circuits for the graph, so that any other circuit can be expressed as a sum of a number of these region boundaries. This fundamental system has the combinatorial property that no edge (or arc) is contained in more than two circuits of the system. This paper is concerned with the converse of this fact: if a combinatorial graph has a fundamental system of circuits such that no edge occurs in more than two circuits, then the graph can be embedded in the plane. The proof of this theorem is largely combinatorial in character. (Received March 13, 1936.)

196. Dr. Saunders MacLane: Note on some equations without affect.

It is possible to use the Eisenstein irreducibility criteria to construct effectively a large class of equations without affect, that is, irreducible equations $f(x)=0$ with rational coefficients which have the symmetric group as Galois group. If $\theta_{1}, \cdots, \theta_{n}$ are the roots of the equation, then $f_{1}(x)=f(x) /\left(x-\theta_{1}\right)=0$ is an equation satisfied by the roots $\theta_{2}, \cdots, \theta_{n}$ after the first root has been adjoined to the rational field. Similarly $f_{i}(x)=f_{i-1}(x) /\left(x-\theta_{i}\right)=0$ would be an equation for $\theta_{i+1}, \cdots, \theta_{n}$ over the field $k_{i}$ containing the first $i$ roots. The group must be the symmetric group if each of these polynomials $f_{i}(x)$ is irreducible over the corresponding $k_{i}$. This can be insured by requiring the original coefficients of $f(x)$ to be divisible by suitable powers of certain primes. These primes can then be decomposed into prime ideals in each $k_{i}$, using Newton polygons, and these prime ideals can be used to show $f_{i}(x)$ irreducible by the Eisenstein criterion. (Received March 13, 1936.)

\section{Dr. W. T. Martin: On expansions in terms of a certain general class of functions.}

The author defines certain generalizations of Appell polynomials and studies the problem of expanding a given function in a series of these generalized functions. The problem of expanding a given function in such a series may also be viewed as a problem of solving a certain functional equation. Existence theorems for these associated functional equations are given. (Received March 19, 1936.)

\section{Mr. A. N. Milgram: Certain extensions of some separation theorems.}

This paper deals with the Brouwer numbers of compact sets as related to those of their sum and intersection. $A_{1}$ and $A_{2}$ are compact subsets of euclidean $n$-space. The relation between the Brouwer numbers of the sets $A_{1}$ and $A_{2}, A_{1} \times A_{2}$, and $A_{1}+A_{2}$ are investigated. Denoting the $r$ th Brouwer number of $A$ by $B^{r}(A)$ we show: (1) if $B^{r-1}\left(A_{1}\right)=B^{r-1}\left(A_{2}\right)=0$ then $B^{r}\left(A_{1}+A_{2}\right)$ $\geqq B^{r-1}\left(A_{1} \times A_{2}\right)$, (2) if $B^{r}\left(A_{1}\right)=B^{r}\left(A_{2}\right)=0$ then $B^{r-1}\left(A_{1} \times A_{2}\right) \geqq B^{r}\left(A_{1}+A_{2}\right)$, and finally (3) if $B^{r}\left(A_{1}\right)=B^{r}\left(A_{2}\right)=0$ and $B^{r-1}\left(A_{1} \times A_{2}\right)-B^{r-1}\left(A_{1}\right)-B^{r-1}\left(A_{2}\right)=m \geqq 0$ then $B^{r}\left(A_{1}+A_{2}\right) \geqq m$. From these theorems one obtains as immediate corollaries the Phragmén-Brouwer theorem, the Janiszewski-Mullikin theorem, and other separation theorems. (Received March 21, 1936.) 


\section{Dr. E. W. Miller: On a property of families of sets.}

It shall be said that a family of infinite sets has property $B$ if there exists a set which contains at least one element of each set of the family but does not exhaust any set of the family. Results in this connection were first obtained by F. Bernstein (Zur Theorie der trigonometrischen Reihe, Berichte der Königlichen Sächsischen Gesellschaft der Wissenschaften, vol. 60 (1908), pp. 325338 ). Bernstein's work shows that any family of sets has property $B$ if the cardinal number of each set of the family is not less than the cardinal number of the family. In the present paper a number of results are obtained for the case where the cardinal number of each set of the family is less than the cardinal number of the family. For instance, it is shown that any non-denumerable family of denumerable sets has property $B$ if each element of each set of the family belongs to at most a denumerable infinity of sets of the family. (Received March 11, 1936.)

\section{Professor Oystein Ore: Direct decompositions.}

This paper contains an analysis of the validity of the main theorem on direct decomposition in algebra by means of the theory of structures. (See the authors' papers, On the foundation of abstract algebra, Annals of Mathematics, vols. 36-37.) (Received March 18, 1936).

\section{Dr. W. C. Randels: On the summability of Fourier series.}

An example is given of a Fourier series which is Abel summable but not $(C, \alpha)$ for any $\alpha$. (Received March 12,1936.)

\section{Dr. M. S. Robertson: On the order of the coefficients of $a$} schlicht function.

If $f(z)=\sum_{1}^{\infty} a_{n} z^{n}, a_{1}=1$, is holomorphic and schlicht for $|z|<1$, then it is known that $\left|a_{n}\right|=O(n)$. It has been conjectured that $\left|a_{n}\right| \leqq n$. It is shown that the functions $f(z)$ which have $\left|a_{n}\right|=\Omega(n)$ are contained only in that class of functions possessing the property that there exists a sequence of points $x_{i}$ inside the unit circle tending to the circumference, and a real number $\alpha$, so that $\lim _{\left|x_{i}\right| \rightarrow 1} F\left(z ; x_{i}\right) \equiv z\left(1+z e^{i \alpha}\right)^{-2}$ where $F\left(z ; x_{i}\right) \equiv\left\{f\left(x_{i}\right)-f\left[\left(x_{i}-z\right) /\left(1-\bar{x}_{i} z\right)\right]\right\}$ $\cdot\left|f^{\prime}\left(x_{i}\right)\right|^{-1} \cdot\left(1-\left|x_{i}\right|^{2}\right)^{-1}$. Failing this property $f(z)$ has coefficients $\left|a_{n}\right|$ $=O\left(n^{1-\delta}\right), \delta=\delta(f)>0$. (Received March 9, 1936.)

203. Dr. M. S. Robertson: On the summability by positive typical means of sequences $f(n \theta)$.

If $\left\{a_{n}\right\}$ is a sequence of non-negative real numbers and $\mu_{n} \equiv \sum_{1}^{n} a_{k}$, some sufficiency conditions are found for $\left\{a_{n}\right\}$ and $f(\theta)$ in order that $M(\theta ; f) \equiv \lim _{n \rightarrow \infty} \mu_{n}^{-1}$ $\cdot \sum_{1}^{n} a_{k} f(k \theta)$ exist and be not greater than $c_{0}=(2 \pi)^{-1} \int_{-\pi}^{\pi} f(\theta) d \theta$ for functions $f(\theta)$ possessing a Fourier series uniformly convergent in $(-\pi, \pi): f(\theta)=\sum_{n=0}^{\infty} b_{n} \sin n \theta$ $+c_{n} \cos n \theta$. With suitable sequences $\left\{a_{n}\right\}$ one obtains the development (uniformly convergent) $M(\theta ; f)=\sum_{n=0}^{\infty} b_{n} M(n \theta ; \sin )+c_{n} M(n \theta ; \cos )$ which will be not greater than $c_{0}$ under suitable conditions. (Received March 9, 1936.) 


\section{Mr. L. B. Robinson: A lacunary function with branch} point at the origin.

This note is the complement of one in the Comptes Rendus, December 23, 1935. Given $u^{\prime}(x)=u\left(x^{3 / 2}\right),\left(u(x) \equiv c_{0}\left\{1+x+(2 / 5) x^{5 / 2}+(2 / 5)(4 / 19) x^{1 / 4}+\right.\right.$ terms calculated by a known law $\}. S_{n}$ is the sum of the first $n$ terms of $u$; it has a branch point at the origin of multiplicity $2^{n-2}$. When $n \rightarrow \infty$ the branches become infinite in number. $u$ converges within the unit circle and on its contour but cannot be prolonged further. One can make similar statements about $u^{\prime}(x)=a(x) u\left(x^{3 / 2}\right)$ where $a(x)$ is an integral function with positive coefficients. (Received March 21, 1936.)

\section{Dr. R. M. Robinson: Bloch functions.}

Bloch's theorem states that there exists an absolute positive constant $P$, such that every function $f(x)=x+\cdots$, which is regular in the unit circle, maps the unit circle on a (many-sheeted) region containing the interior of a circle of radius $P$ (in a single sheet). If $\mathfrak{B}$ represents the upper bound of such constants $P$, it is shown that every function $f(x)=x+\cdots$, which is regular in the unit circle, and maps the unit circle on a region not containing the interior of any circle of radius $>\mathfrak{B}$ in a single sheet, has the unit circle as a natural boundary. (Received March 9,1936.)

206. Mr. Benjamin Rosenbaum: On products of successive terms of an arithmetic series.

Let $H=$ the index of the highest power of the prime $p$ dividing $\prod_{x=0}^{n-1}\left(x a+c_{0}\right)$, where $a$ and $c_{0}$ are relatively prime positive integers, and $a$ is not divisible by $p$. Let $i_{1} a+c_{0}=c_{1} p$, where $i_{1}$ is the smallest integer $\geqq 0$ such that $i_{1} a+c_{0}$ is divisible by $p$. Similarly let $i_{r} a+c_{r-1}=c_{r} p$. Simple formulas are obtained for $H$ involving respectively: (1) the integers $i$; (2) the integers $c$; (3) the digits of $n$ to the base $p$; (4) the digits of $a(n-1)+c_{0}$ to the base $p$. Limiting values for $H$ are also obtained. The formulas are easily modified for the general case where $a$ and $c_{0}$ are positive integers with no other restrictions. The above results are an extension, with certain corrections, of the work of Carmichael (this Bulletin, vol. 15 (1908-1909), pp. 217-221). (Received March 4, 1936.)

207. Professor I. J. Schoenberg: A number theory problem concerning regular simplexes.

It is well known and usually proposed as a problem in elementary texts that in the plane of a rectangular coordinate system there is no equilateral triangle whose vertices have integral coefficients only. This can be stated as follows: One cannot inscribe a regular 2-dim. simplex $S_{2}$ in the 2-dim. regular lattice $L_{2}$. As readily seen, it is always possible to inscribe an $S_{m}$ (m-dim. regular simplex) in $L_{n}$ ( $n$-dim. regular lattice) if $m<n$, while this is obviously impossible if $m>n$. The only case of interest is therefore $m=n$. The following theorem is proved: (i) If $n$ is even it is possible to inscribe an $S_{n}$ in $L_{n}$ if and only if $n+1$ is a square. (ii) If $n$ is odd, this construction is always possible if $n \equiv 3(\bmod 4)$; if, however, $n \equiv 1(\bmod 4)$, then the construction is possible if and only if $n+1$ is not divisible to an odd exponent by a prime number of the form $4 k+3$. Thus 
one can inscribe an $S_{n}$ in $L_{n}$ for $n=3,7,8,9,11,15,17,19,23,24,25,27,31$, $33, \ldots$ and not for the numbers left out. It is of interest to point out that the case when $n$ is even can be treated completely by a very elementary geometric method, while the case of an odd $n$ requires a great deal of Minkowski's work in the arithmetical theory of quadratic forms of $n$ variables, in particular the theory of the equivalence of such forms with respect to linear transformations with rational coefficients (Gesammelte Abhandlungen, vol. 1, pp. 219-239). (Received March 18, 1936.)

\section{Professor I. J. Schoenberg: On certain two-point expan-} sions of integral functions of exponential type.

The interpolation problems (1) $f^{(2 n)}(1)=a_{n}, f^{(2 n)}(0)=b_{n},(n=0,1,2, \cdots)$, and $(2) f^{(2 n)}(1)=a_{n}, f^{(2 n+1)}(0)=b_{n},(n=0,1,2, \cdots)$, to be solved by integral functions $f(x)$, were investigated by Hillel Poritsky (Transactions of this Society, vol. 34 (1932), pp. 274-331) and J. M. Whittaker (Proceedings London Mathematical Society, vol. 36 (1933-34), pp. 451-469). Here the author solves the unicity questions concerning these interpolation problems for functions of exponential type. They are answered by the following two theorems. I. The only functions of exponential type satisfying the system $f^{(2 n)}(1)=0, f^{(2 n)}(0)=0$, $(n \geqq 0)$, are the sine polynomials $f(x)=\sum_{\nu=1}^{n} c_{\nu} \sin \nu \pi x$ with arbitrary coefficients. II. The only functions of exponential type satisfying the system $f^{(2 n)}(1)=0, f^{(2 n+1)}(0)=0,(n \geqq 0)$, are the cosine polynomials given by the formula $f(x)=\sum_{\nu=0}^{k} c_{\nu} \cos (2 \nu+1) \pi x / 2$. These results are readily proved by means of a theorem of R. D. Carmichael. (Received March 21, 1936.)

\section{Dr. Wladimir Seidel: On the order of growth of univalent functions.}

Let $f(z)$ be a regular univalent function in the unit circle $|z|<1$. Let $\theta=\theta_{j}(j=1,2, \cdots, n)$ be $n$ radii on which $f(z)$ satisfies the condition $0<k$ $<\left|f\left(r e^{i \theta j}\right)\right| /[1 /(1-r)]^{\alpha_{j}}$ for some constant $k$ and $n$ positive constants $\alpha_{j}$. If $\sum_{j=1}^{n} \alpha_{j}=2$, then on every radius, other than the radii $\theta=\theta_{j}$, the function $f(z)$ satisfies the relation $\lim _{r \rightarrow 1} f\left(r e^{i \theta}\right) /[1 /(1-r)]^{\delta}=0$ for every constant $\delta>0$. An example is given to show that the conclusion can not be replaced by the stronger one of boundedness. Further results are obtained on the order of growth of the derivative of a univalent function. (Received March 14, 1936.)

\section{Professor I. M. Sheffer: A characterization of a class of polynomials.}

Pincherle was perhaps the first to point out the value of Appell polynomials in the study of certain linear functional equations. There are other sets of polynomials that serve a similar purpose, and the author here considers a class of such polynomials. If $J$ is a suitable linear operator, polynomials $\left\{P_{n}(x)\right\}$ satisfying the relation $J\left[P_{n}(x)\right]=P_{n-1}(x)$ are considered. A characterization of such polynomials is obtained, and an examination made of the functional equations satisfied by these polynomials. (Received March 11, 1936.) 


\section{Dr. C. G. Shover: Number theory in a hypercomplex system.}

The maximal domains of integrity of the division algebras used in the matric representation of a semi-simple system determine the maximal domains of the latter. The author employs Artin's definition of a maximal domain (Abhandlungen mathematisches Seminar Hamburg Universität, vol. 5 (1928), pp. 261-289). Likewise, there is a biunique correspondence between the twosided integral ideals of an integral ring of the automorphic field of a simple onesided ideal and those of the corresponding maximal domain of integrity of the system. The two ideas of operator isomorphism and equivalence are proved to be identical for non-singular ideals. On this basis it is proved that the twosided class number of a system is equal to that of the division algebra and the one-sided class number is determined by the one-sided class number in the division algebra and the number of simple left (or right) ideals in the system. (Received March 11, 1936.)

\section{Dr. Abraham Sinkov: On a perfect group of order 10,752.}

The defining relations $S^{3}=T^{2}=(S T)^{7}=\left(S^{-1} T^{-1} S T\right)^{p}=1$ abbreviated $(2,3,7 ; p)$, have been studied for all values of $p$ up to and including seven. In each case, the four conditions have been shown to be either incompatible or else sufficient to define a single finite group. This does not appear to be true when $p$ exceeds seven; it seems probable that more than one group exists satisfying the relations $(2,3,7 ; p), p>7$. If that be true, then there exist certain combinations of the generators whose periods are independent of the four initial relations. The present paper selects one such combination and obtains some general restrictions on its possible periods. With the aid of these results, it is proved that $G^{3,7,11}$ collapses. It is also shown that the simple group of order 168 and a perfect group of order 10,752 are both factor groups of $(2,3,7 ; 8)$. (Received March 10, 1936.)

\section{Mr. N. E. Steenrod: Universal homology groups.}

The Čech homology groups of a topological space $F$ over a topological abelian group of coefficients are topological groups. The same is true of the homology groups of the infinite cycles of an infinite complex $K$. These groups of $K$ always decompose into the direct sum of a torsion group and a reduced homology group. The homology groups of $K$ over the real numbers mod 1 form a complete set of invariants for the class of all homology groups of $K$. To each compact metric $F$ corresponds a $K$ whose homology groups are those of $F$ of one less dimension. The above theorems therefore hold for $F$. This verifies a surmise of P. Alexandroff (Annals of Mathematics, vol. 36 (1935), p. 34). A mod 1 homology group of $F$ of dimension $>1$ does not in general have its component of zero as a direct summand. A bicompact connected abelian group is continuously isomorphic with its 1-dimensional homology group mod 1. Much of the argument is based on the character group theory of Pontrjagin. (Received March 10, 1936.) 
214. Professor Gabriel Szegö: On some hermitian forms associated with two given curves of the complex plane.

The smallest characteristic value of Hilbert's quadratic form $\sum(\mu+\nu+1)^{-1} x_{\mu} x_{\nu}(\mu, \nu=0,1,2, \cdots, n)$ can be represented asymptotically as follows: $2^{15 / 4} \pi^{3 / 2} n^{1 / 2}\left(2^{1 / 2}-1\right)^{4 n+4}$, if $n \rightarrow \infty$. This is a special case of the general problem of determining the maximum of the square integral of all polynomials of the $n$th degree on a given curve, provided that the corresponding square integral on another curve is prescribed. Some cases of infinite integrals are considered from a similar point of view. For instance, the smallest characteristic value of $\sum(\mu+\nu) ! x_{\mu} x_{\nu}(\mu, \nu=0,1,2, \cdots, n)$ is asymptotically $2^{3 / 2} \pi^{3 / 2} e n^{1 / 4} e^{-4(n)^{1 / 2}}$, if $n \rightarrow \infty$. The investigation makes use of the asymptotic formula of Legendre and related polynomials exterior to the orthogonality interval. (Received March 21, 1936.)

215. Mr. W. S. Turpin: Concerning special centers of projection for an algebraic space branch.

Given an algebraic branch $\Gamma$ in $S_{3}$, its projection, $\Gamma_{1}$, from a point $O$ onto a plane will have a certain composition when $O$ is a generic point of $S_{3}$. For special centers of projection, $\tilde{O}$, this composition will be altered. It is found that the locus of these special centers consists of a set of planes passing through the tangent line of the space branch. These planes are explicitly determined in terms of the coefficients of the expansion of the space branch. The osculating plane of the branch is not necessarily contained in this set. (Received February 29, 1936.)

216. Mr. W. S. Turpin: On the fundamental group of a certain class of plane algebraic curves.

Let $C$ denote the plane algebraic curve $\Phi^{2}+\Psi^{3}=0$ where $\Phi$ and $\Psi$ are general polynomials, arbitrarily selected, in $x$ and $y$ of respective degrees $3 m$ and $2 m$, and $m$ is any positive integer. Let $G$ be the Poincaré group of the residual space of the curve $C$ relative to its carrying complex projective plane. It is found that $G$ is generated by two elements, $U$ and $V$, satisfying the relations: $U^{2}=V^{3}$; $U^{2 m}=V^{3 m}=1$. The proof of this result is divided into two parts: (1) degeneration of $C$ into a limit curve, $\bar{C}$, on which several singularities, distinct on $C$, have become coincident and whose fundamental group is easily ascertained; (2) careful reconstruction of the individual generating relations of $G$ which are lost due to coincidence of singularities on $\bar{C}$. (Received February 29,1936.)

\section{Dr. H. E. Vaughan: On the convergence of manifolds and irreducible membranes.}

This paper makes application of the notion, introduced by G. T. Whyburn (Fundamenta Mathematicae, vol. 25 (1936), pp. 408-426), of regular convergence of a sequence of sets. The following theorems are demonstrated: 1. Let $\left(K_{i}\right)$ be a sequence of closed subsets of a compact metric space which converges regularly relative to $m$-cycles, $0 \leqq m \leqq n$, to the limit $K$. Suppose also that $K_{i}$ is an irreducible membrane relative to a complete $n$-cycle and that there 
exists a sequence of carriers of these cycles whose limit is a proper subset of $K$. Then $K$ is an irreducible membrane with respect to a complete $n$-cycle. 2 . Let $\left(M_{i}\right)$ be a sequence of 2 -manifolds which converges regularly relative to 0 and 1-cycles to the non-degenerate limit $M$. Then $M$ is a 2-manifold. (Received March 14, 1936.)

\section{Dr. J. F. Wardwell: Non-separating transformations.}

If $A$ is a compact continuum, a single-valued transformation $T(A)=B$ will be called non-separating provided that no set $T^{-1}(b), b \in B$, separates $A$. Such a transformation is equivalent to an upper semi-continuous decomposition of $A$ into sets which do not separate $A$. Every such transformation is obviously non-alternating (see G. T. Whyburn, American Journal of Mathematics, vol. 56 (1934), pp. 294-302). In this paper non-separating transformations are studied and various results are obtained. In particular it is shown that if $T(A)=B$ is non-separating, $B$ will contain no cut points. Hence, if $A$ is locally connected, $B$ will be cyclicly connected. It is also shown that, in this case, there will exist a unique cyclic element $E_{a}$ of $A$ such that $T\left(E_{a}\right)=B$. Results of the application of such transformations to particular curves and surfaces, such as simple closed curves, boundary curves, spheres, and cactoids, are obtained. (Received March 6, 1936.)

219. Dr. S. E. Warschawski: On the behavior of a conformal mapping at a cusp.

Let $C$ be a closed Jordan curve in the w-plane with a cusp at $w=0$, the two semi-tangents being on the positive real axis; that is, in a neighborhood of $w=0, C$ consists of two branches $C_{+}$and $C_{-}$, represented by the equations $\phi=\phi_{+}(r), \quad \phi=\phi_{-}(r), 0<r \leqq a$, respectively $\left(\phi_{+}(r)>\phi_{-}(r)\right)$, with $\lim _{r \rightarrow 0} \phi_{+}(r)$ $=\lim _{r \rightarrow 0} \phi_{-}(r)=0(r, \phi$ polar coordinates, center at $w=0$, the angle $\phi$ is measured from the positive real axis). Moreover, it is assumed that $C_{+}$and $C_{-}$satisfy the "Lindelöf condition": the slope of any chord $A B$ of $C_{+}$or of $C_{-}$approaches 0 as $A$ and $B$ approach $w=0$ simultaneously. Let $z=z(w),(z(0)=0)$, map $|z+1|<1$ conformally on the interior $R$ of $C$. The order of magnitude of $z(w)$ as $w \rightarrow 0$ is studied and results like the following are obtained: Let $\phi(r)=\phi_{+}(r)$ $-\phi_{-}(r)$, and $|w|=r$. Then: (a) $\lim _{r \rightarrow 0}[\log |z(w)|] / \int_{r}^{a} d \rho /(\rho \phi(\rho))=-\pi$, for $w$ in $R+C ;$ (b) $\lim _{r \rightarrow 0}\left|z^{\prime}(w) / z(w)\right| r \phi(r)=\pi$ in any sub-region $\{0 \leqq r \leqq a$, $\phi=t \phi_{+}(r)+(1-t) \phi_{-}(r), 0<\delta \leqq t \leqq 1-\delta, \delta$ fixed $\}$ of $R$; (c) $\lim _{r \rightarrow 0}|z(w)| / \exp$ $\left[-\pi \int_{r}^{a} d \rho /(\rho \phi(\rho))\right]=\lambda$ exists always $(w$ in $R+C)$ and $0 \leqq \lambda<\infty ;(\mathrm{d})$ criteria are given for $\lambda$ being positive. (Received March 14, 1936.)

\section{Professor Hassler Whitney: The maps of a 4-complex into a 2-sphere.}

The maps of an $m$-complex $K^{m}$ into an $m$-sphere $S^{m}$ have been classified, but not those into $S^{n}$ for $n<m$. Take $m=4, n=2$. Let $K^{i}$ be the $i$-dimensional part of $K^{4}$. A map of $K^{4}$ into $S^{2}$ determines a 2-chain $A^{2}$ in $K^{4}$, which is a "contracycle" if and only if the map can be extended over $K^{3}$. The map can be extended over $K^{4}$ if and only if the "product" (Čech-Whitney) $B^{4}$ of $A^{2}$ with itself vanishes. The classes of maps of $K^{3}$ into $S^{2}$ are in $(1-1)$ corre- 
spondence with the elements (determined by $A^{2}$ ) of the "2-contrahomology group" of $K^{3}$ (compare abstract 41-11-376) times the elements of the difference group of 3-contracycles minus all 2-contraboundaries and minus all products of 1-contracycles with $A^{2}$. The coefficient group in the above is the group of integers. The maps of $K^{4}$ into $S^{2}$ are partially classified. (Received March 14, 1936.)

221. Dr. Max Zorn: Absolute full reducibility of semi-simple distributive linear algebras.

The paper studies the behaviour of semi-simple linear algebras under extension of the reference field. No associativity relations are required. (Received March 13, 1936.)

\section{Dr. Max Zorn: Galois theory based on the lemmata of} Artin and Baer.

This paper contains an analysis and simplification of the Galois theory for finite and infinite normal extensions. (Received March 13,1936.)

223. Dr. Max Zorn: On a formal decomposition theorem for algebras.

Artin has given an equivalence definition for ideals in associative and other types of algebras. This paper gives an equivalence analogue of the full reducibility theorem for algebras without a radical. (Received March 13,1936.)

224. Dr. Max Zorn: On the principles of d'Alembert, Jourdain, and Gauss.

The paper gives an analysis of the differential principles in classical mechanics. It is shown that the inversion of the principles suggests a simple definition of constraints without friction. This definition, following ideas of Budde, allows a simple arrangement of the proofs. (Received March 13,1936.)

\section{Dr. L. V. Ahlfors: On Phragmén-Lindelöf's principle.}

For functions analytic in a half-plane and bounded on the imaginary axis a convex property, analogous to Hadamard's three circles theorem, is proved. From this property the asymptotic statement known as Phragmén-Lindelöf's principle, in the precise form due to the brothers Nevanlinna, is seen to follow by geometric inspection. The method can be extended to harmonic functions of $n$ variables. (Received April 6, 1936.)

226. Professor E. S. Akeley: Rational tensor functions of the coefficients of a pair of quadratic differential forms and their first derivatives. Preliminary report.

This paper is a continuation of the work described in abstract 42-5-227. The non-degenerate case is considered where the $\lambda_{m}$ are all unequal. Define $k_{m}=(1 / h)\left[\partial\left(h h_{m}^{\alpha}\right) / \partial x^{\alpha}\right](m)$. Each $k_{m}$ is invariant under coordinate transformations, but changes sign under the reflections $h_{m} \rightarrow \pm h_{m}{ }^{\prime}$. Assume that $k_{m} \neq 0(m)$. Then $k_{m} h_{m}(m)$ represent a system of $n$ orthogonal vectors, uniquely determined 
by the two forms. Also $V_{r}=\sum_{m}^{1-n} e_{m} \lambda_{m}^{m} k_{m} h_{m}$ is a system of $n$ linearly independent vectors, which are invariant under the group $G$, and which are therefore rational functions of the coefficients of the two forms and their first derivatives. This system of vectors can be orthogonalized if desired. Any tensor invariant under $G$ can then be expressed uniquely in terms of either of these systems and the rational invariants of the two forms and their first derivatives. The general form of such invariants when expressed in terms of the $\lambda_{m}, h_{m \alpha}$ has been determined. The general case where the $\lambda_{m}$ are not all different is still being investigated. Invariants and vectors corresponding to the $k_{m}$ and $k_{m} h_{m}$ are only associated with $\lambda_{m}$ of multiplicity unity. (Received March 27,1936.)

\section{Professor E. S. Akeley: Tensors associated with a pair of quadratic differential forms.}

Given a pair of forms $g_{\alpha \beta} d x^{\alpha} d x^{\beta}, G_{\alpha \beta} d x^{\alpha} d x^{\beta}(\alpha, \beta=1, \cdots, n)$ that can be represented thus $g_{\alpha \beta}=\sum_{m}^{1-n} e_{m} h_{m \alpha} h_{m \beta}, G_{\alpha \beta}=\sum_{m}^{1-n} e_{m} \lambda_{m} h_{m \alpha} h_{m \beta}$ (1). Certain tensors associated with the forms can be expressed much more simply in terms of the auxiliary quantities, viz. the invariants $\lambda_{m}$ and the vectors $h_{m}$ with components $h_{m \alpha}$. The following theorem is a consequence of the Galois theory: The class of all rational tensor functions of the $g_{\alpha \beta} G_{\alpha \beta}$ and their higher derivatives up to the $r$ th order is identical with the class of all rational tensor functions of the auxiliary quantities and their higher derivatives up to the $r$ th order, which are invariant under the Galois group of transformations that leave the relations (1) invariant. The computation of such tensors expressed as functions of the auxiliary quantities and their higher derivatives in terms of the $g_{\alpha \beta} G_{\alpha \beta}$ is reduced to the computation of certain symmetric functions of the $\lambda_{m}$. Every rational tensor function of the $g_{\alpha \beta}$ and $G_{\alpha \beta}$ can be expressed in terms of a fundamental system of tensors, each tensor of the system being invariant under $G$. This system can be orthogonalized. The above forms a generalization of the elementary theory of symmetric functions. (Received March 27, 1936.)

\section{Mr. Garrett Birkhoff: Continuous free groups.}

To each integer $n$ corresponds a "continuous free group" $G_{n}$ with the following properties. $G_{n}$ is a topological group. $G_{n}$ has canonical parameters mapping it in the large onto the linear space $(s)$. Under these parameters the function of composition of $G_{n}$ is the sum of an infinite series of polynomials, and any topological automorphism of $G_{n}$ is a linear transformation. $G_{n}$ has a Lie algebra, containing the "free" Lie algebra generated by $n$ symbols as a dense subalgebra. One can assign to every rectifiable path $X$ issuing from the origin in $n$-space $E_{n}$, a "value" $v(X) \epsilon G_{n}$. The $v(X)$ are an everywhere dense set; hence by "completing" (in the topologico-algebraic sense of van Dantzig) a suitably topologized path-group, one obtains another definition of $G_{n}$. This path-group is significant in geometry and differential equations. One can also define $G_{n}$ from the discrete free group $D_{n}$ generated by $n$ symbols by iterated dyadic interpolation, passage to the limit, and "completion." (Received March 25, 1936.)

229. Professors Einar Hille and Otto Szász: On the completeness of Lambert functions. II.

In this paper the authors continue their study of the completeness prop- 
erties in $L_{1}(0,1)$ of sets like $\left\{\phi(t) \sum_{m-1}^{\infty} a_{m} t^{m \lambda_{n}}\right\}$ for arbitrary weight functions $\phi(t)$, and coefficients $a_{m}$ such that $a_{1}=1,\left|a_{m}\right| \leqq B m^{\beta}$. The method extends to the case of irregular power series whose exponents form a multiplicative system. They also prove the completeness in $C[0,1]$ of certain special systems of Lambert functions with $\phi(t)=(1-t)^{\beta+1}, a_{m}=m^{\beta}, \lambda_{n}=n$, for $\beta=0,1$, and 2. (Received April 6, 1936.)

230. Professor Marston Morse: Functional topology and abstract variational theory.

The critical reader will find, in certain of the recent attempts to extend the results of the author on functionals, serious deficiencies of two kinds. Either the definitions involved are not sufficiently inclusive, or they are so formulated that the desired inequalities do not hold. Moreover, much irrelevant material has been introduced. The author has found that the problem is structurally a group theory problem. If one replaces the comparisons implied by the finite counting of type numbers by the comparisons of certain basic groups $M_{k}$ of relative $k$-cycles under suitably chosen isomorphisms naturally defined by $F$ and termed ${ }^{*}$-isomorphisms, the theory is immediately enlarged and simplified. The $(n+1)$ st fundamental numerical inequality is now replaced by a group theory relation of the form $M_{n} \bmod ^{*} M_{n-1} \bmod ^{*} M_{n-2} \cdots \bmod ^{*} M_{0} \geqq 0$ $(n=0,1, \cdots)$ where $\geqq 0$ can be read "exists" and $\bmod ^{*}()$ is to be read, $\bmod$ a suitably chosen group ${ }^{*}$-isomorphic with ( ). In the old inequalities $\bmod ^{*}$ was alternatingly plus and minus and $M_{k}$, as it now appears, was the dimension of a finite group. The underlying space is metric and the function lower semicontinuous. (Received April 4, 1936.)

231. Professor L. E. Dickson: Proof of the ideal Waring theorem for exponents exceeding six.

(Received March 13, 1936.)

\section{Professor L. E. Dickson: Asymptotic Waring theorems.}

Let $k \leqq n\left(6 \log _{e} n+\log _{e} 216+4\right)-2$. Let $\log _{10} N=2 n^{7}$. If $n \geqq 9$, every integer $\geqq N$ is a sum of $4 n-2+6 k$ integral $n$th powers $\geqq 0$. This Waring constant $N$ is far smaller than that found by the various earlier methods. Its smallness is essential for the companion paper on the ideal Waring theorem. (Received March 13, 1936.)

\section{Professor W. J. Trjitzinsky: Concerning linear difference equations containing a parameter.}

This paper gives the extension to a more general problem of the results and methods of an earlier paper by the author on the subject of linear difference equations containing a parameter. (Annali di Matematica, series 4, vol. 15 (1936), pp. 181-214.) (Received March 30, 1936.)

\section{Professor Morris Marden: A note on critical points.}

Professor Walsh has recently proved some theorems about the critical points of Green's function $G(x, y)$ (pole at $\infty$ ) for an infinite region with an 
arbitrary finite boundary $B$, and of a function harmonic in a region bounded by two sets of arbitrary Jordan curves $C_{1}$ and $C_{2}$ and equal to zero on $C_{1}$ and one on $C_{2}$. The method he uses is that of approximation by lemniscates, but he also suggests that of forces attracting according to the inverse distance law. The latter, identical with the method of partial fractions, seems easier in the restricted case that $B$ consists of regular curves. The formulas then needed may be derived from Walsh's formulas or from Cauchy's Integral Theorem. Among the results thus obtained is the following: If $K$ is any convex region enclosing $B$, then at no point where $K$ subtends an angle less than $\pi / k$ can all the $k$ th partial derivatives of $G(x, y)$ simultaneously vanish. (Received April 10, 1936.)

\section{Mr. Archie Higdon and Professor D. L. Holl : Stresses in moderately thick rectangular plates.}

The displacements $(u, v, w)$ are expressed as power series $\sum_{0}^{\infty}\left(U_{n}, V_{n}, W_{n}\right) z^{n}$, where the coefficients of $z^{n}$ are functions of $(x, y)$. To satisfy the equilibrium equations, one may show by the use of recurrence relations that the displacements are expressible in terms of $U_{0}, U_{1}, V_{0}, V_{1}, W_{0}$, and $W_{1}$. Employing a method using orders of magnitude (Sibert, Transactions of this Society, vol. 33 (1931), pp. 329-370), a solution is obtained which is made to satisfy the surface loads on the faces of the plate. In particular for a normal hydrostatic load $p(x, y)=p_{0}\left(1+\alpha x a^{-1}+\beta y b^{-1}\right)$, solutions are effected for three types of edge conditions; pinned-pinned, pinned-free, and pinned-clamped edges. When $\alpha=\beta=0$, the known solutions of Garabedian (Comptes Rendus de l'Académie des Sciences, vol. 195 (1932), pp. 1369-1371) are included. Since the lowest order of magnitude in $W_{0}$ is the corresponding thin plate solution, these solutions, the last two of which have not hitherto been recorded, are given explicitly. The method is applicable to any type of load which is a polynomial in $(x, y)$ and continuous over the entire surface of the plate. (Received April 11, 1936.)

236. Professor J. L. Walsh and Mr. W. E. Sewell: Sufficient conditions for various degrees of convergence by polynomials.

Let $C$ be an arbitrary closed limited point set in the z-plane, whose complement $K$ is connected and regular in the sense that $K$ possesses a Green's function $G(x, y)$ with pole at infinity. Let $C_{R}$ denote generically the locus $G(x, y)$ $=\log R>0$. If the function $f(z)$ is expressible interior to $C_{R}$ by the Cauchy integral over $C_{R}$, if $p_{n}(z)$ is an arbitrary polynomial of degree not greater than $n$, and if the points $z_{1}, z_{2}, \cdots, z_{n+1}$, lie interior to $C_{R}$ and $\omega_{n}(z)=\left(z-z_{1}\right)\left(z-z_{2}\right)$ $\cdots\left(z-z_{n+1}\right)$, then one has, for $z$ interior to $C_{R}$, (1) $f(z)-P_{n}(z)=(1 / 2 \pi i) \int_{C_{R}}[f(t)$ $\left.-p_{n}(t)\right] \omega_{n}(z) d t /\left[\omega_{n}(t)(t-z)\right]$, where $P_{n}(z)$ is the polynomial of degree $n$ interpolating to $f(z)$ in the points $z_{k}, k=1,2, \cdots, n+1$. Continuity properties of $f(z)$ on $C_{R}$ yield (S. Bernstein, de la Vallée Poussin, D. Jackson, Sewell) inequalities for $\left|f(z)-p_{n}(z)\right|$ on $C_{R}$, or for $\int_{C_{R}}\left|f(t)-p_{n}(t)\right| p|d t|$. By (1) these inequalities are then used to obtain results on the degree of convergence of $P_{n}(z)$ to $f(z)$ on $C$. (Received March 30,1936.) 


\section{Professor John von Neumann: On the uniqueness of invariant Lebesgue measures.}

A. Haar proved in 1933 the existence of a right-invariant Lebesgue measure in every locally compact topological group. The uniqueness of his measure, however, was not obvious. It was established for compact groups by the author in 1934, and generally for all locally compact groups by A. Weil and the author in 1935. A simpler proof of this is given, which is also more general. First this problem is considered: Given a space $S$ and a transitive group $G$ of transformations of $S$. How many Lebesgue-measures in $S$ exist, for which the transformations of $G$ carry the sets of measure 0 into each other? The result is: If a rightinvariant Lebesgue measure exists in $G$, then such measures exist in $S$, and they all have the same 0 -sets. This is obtained without topological assumptions, by the use of Borel sets only, and assuming only that the composition-laws in $G$ and $S$ are described by Baire-functions. The proof obtains by application of Fubini's theorem. The uniqueness-theorem for right-invariant Lebesguemeasures follows from the above result by considering the decompositionproperties of non-0-sets. (Received April 23, 1936.)

238. Dr. J. B. Rosser (National Research Fellow): Rational proofs of the existence of primes in arithmetic sequences.

It is proved that for each pair of positive integers, $k$ and $l$, which are relatively prime, there exists a direct rational proof that there is an infinity of primes of the form $k \alpha+l$. In describing these proofs as "direct rational proofs," it is meant that they are the type of proof that every one would agree to be direct and elementary. To be more explicit, reductio ad absurdum is not used, and real numbers and limiting processes are not used, the argument being carried on wholly by means of simple properties of $+, X,<$, and raising to integer powers as applied to rational numbers, and by means of mathematical induction as applied to positive integers. As the rational proofs vary with $k$ and $l$, and the proof that the rational proofs exist is transcendental, this theorem does not afford the means of inferring the existence of a single direct rational proof that holds for all $k$ and $l$. (Received April 20,1936.)

239. Professor Jesse Douglas: Minimal surfaces of general topological structure with any finite number of assigned boundaries.

This work goes back to the author's paper of nearly seven years ago, $A$ general formulation of the problem of Plateau, presented by title to the Society in October, 1929, an abstract of which was published in this Bulletin, vol. 36, p. 50. The solution of the problem there formulated for the first time was described by the author in a recently published paper, Some new results in the problem of Plateau, Journal of Mathematics and Physics, vol. 15, no. 1 (Feb. 1936), pp. 55-64. The present paper is an elaboration of this one, giving proofs and other details. Included is an example of a contour bounding a minimal surface of the topological form of a torus with one perforation. This contour is formed by two nearby parallels and two nearby meridians of a torus, when the curvilinear square which they form by their intersection is removed. It was first given by the author in his invited address to the Society on the 
Plateau problem in October, 1932, but has not hitherto been printed. (Received April 28, 1936.)

\section{Mr. Garrett Birkhoff: Lie groups isomorphic in the large} with no linear groups.

Hitherto no example has been known of a Lie group which can be proved to be isomorphic with no group of matrices. This note shows that every noncommutative finite continuous group whose Lie algebra is nilpotent is locally isomorphic with at least one Lie group which is topologically isomorphic in the large with no group of matrices. (Received April 29, 1936.)

\section{Mr. Garrett Birkhoff: Free continuous groups. II.}

The author is able to deduce many corollaries from the theorem (proved transcendentally) that the "free Lie algebra" $L_{r}$ consisting of linear forms in (non-commutative, non-associative) products of symbols $x_{1}, \cdots, x_{r}$, modulo the identities $[u v]+[v u]=0$ and $[[u v] w]+[[v w] u]+[[w u] v]=0$, is isomorphic with the subsystem of the free (non-commutative) associative hypercomplex algebra generated by the $x_{i}$, determined by the operation $[u v]=u v$ $-v u$. One corollary is that the central of the quotient-group of the free discrete group generated by $r$ symbols, over the characteristic subgroup generated by commutators of "weight" $\geqq n$ (in the sense of P. Hall), is the subgroup generated by commutators of "weight" $\geqq n-1$. (Received April 29, 1936).

242. Professor J. C. Trainor: Problems on the borderline between mathematics and psychology.

The close relationship between mathematics and the physical sciences has yielded great benefit to both. There is urgent need for a similar relationship between mathematics and the human sciences, particularly psychology. At present there are two significant approaches to the problems involved in using mathematical frameworks for psychological data: (1) the development of the factor-vector methods of Spearman, Thomson, Kelley, Thurstone, Williams, and others. Here the mathematical formulation is rigorous but the data often suspect; (2) the General Semantics theory of A. Korzybski which offers a satisfactory comprehensiveness but has not an explicit mathematical formulation. Psychologists can train themselves to be mathematicians or mathematicians can master the literature of psychology. The latter approach would seem more practicable for the purpose of furthering the immediate attack on the many problems which are borderline to the two fields. (Received April 20, 1936.)

243. Mr. Alvin Sugar: A cubic analogue of the Cauchy-Fermat theorem. II.

By considering the cases $5<m<16$ and combining this with known results, we obtain the following theorem: Every positive integer is a sum of $m+3$ values of the polynomial $P(x)=m\left(x^{3}-x\right) / 6+x$ for $m \geqq 6$ and is a sum of nine values of $P(x)$ for $0<m<6$. For every $m \geqq 6$ this result gives us an ideal universal Waring theorem. (Received April 25, 1936.) 
244. Professor F. A. Lewis: Proof of the non-isomorphism of two collineation groups of order 5184 .

Musselman has treated (American Journal of Mathematics, vol. 49 (1927), no. 3) a collineation group $G$ of order 5184 which belongs to a class of groups mentioned by Winger (ibid, vol. 52, (1930), p. 394). In this paper the author defines a collineation group $C_{6}$ of degree 6 and order 5184, which also belongs to an infinite class of collineation groups, and proves $G$ and $C_{6}$ are not simply isomorphic. The method of proof consists in showing that the number of collineations of period 2 in $G$ exceeds the number of collineations of period 2 in $C_{6}$. (Received April 27, 1936.)

245. Professor Morgan Ward: Linear divisibility sequences.

Let $(u): u_{0}=0, u_{1}=1, u_{2}, \cdots, u_{n}, \cdots$ be a linear recurring series of rational integers whose associated polynomial $f(x)=x^{k}-c_{1} x^{k-1}-\cdots-c_{k}$ has no repeated roots and co-prime integral coefficients. We assume that $(u)$ is a divisibility sequence: $u_{n}$ divides $u_{m}$ if $n$ divides $m$. The series $(u)$ will be said to be primary if there exists no integer $>1$ dividing all terms of $(u)$ beyond a certain point. We prove: 1 . If $(u)$ is primary, then for all large primes $q, u_{q}^{t} \equiv 1$ $(\bmod q)$ where $t$ is the least common multiple of $1,2, \cdots, k$. 2. If $(u)$ is not primary, it always contains an infinite number of subsequences which are primary. 3. There exists a rational number $B$ depending only on the initial values of the sequence and upon the recurrence such that the rank of apparition of any prime $p$ in $(u)$ which divides neither the numerator nor denominator of $B$ is the restricted period of $(u)$ modulo $p$. The prime factors of the denominator of $B$ all divide the discriminant of $f(x)$, and $B$ can never vanish if the Galois group of $f(x)$ is alternating or symmetric. (Received May 1, 1936.)

\section{Professor A. F. Carpenter: Self-corresponding line com- plexes.}

By means of a certain pair of quadrics associated with each line element of the general ruled surface $R$, this paper sets up a correspondence, to each line of space, of a linear complex in such a way that when this correspondence is extended over all the line elements of $R$, all the linear complexes of space are accounted for. Among these there are shown to exist certain invariant complexes. (Received May 2, 1936.)

247. Professor A. A. Shaw: On measures and weights by Epiphanius.

The purpose of this study is to issue an English translation with critical commentary, from the Venice edition (1821) published by the Mukhtharist Order of St. Lazar, Venice, as no English translation exists of this unique treatise. It will include: (1) an introduction giving an account of the life and works of Epiphanius; (2) translation of the text pointing out in foot notes, variant readings from the text of $P$. de Lagarde, or doubtful passages; (3) notes which will explain, among other things, all the technical terms in terms of modern units; for purposes of calculation, the editor has found it convenient to adopt the Roman-Byzantine litra (326.4 gms) and the Roman xestes (408 
gms) as his fundamental units; (4) a Greek-English vocabulary of the text; (5) the Greek text; and (6) a bibliography. Since frequent reference is made in this work to the Weights and Measures of Ananiah Shiragooni, some account is also given of the latter in the form of an appendix. (Received May 2,1936.)

\section{Professor R. E. Moritz: Note on Taylor's theorem.}

The proof of Taylor's theorem, which has found its way into the great majority of American textbooks on the calculus, dates back to Homersham Cox. While admittedly the most satisfactory of all existing proofs of the theorem, it is open to the charge of artificiality inasmuch as it makes use of an auxiliary function which does not naturally suggest itself. The author's note shows how the auxiliary function may be avoided and the proof of the theorem shortened without any other assumptions except those of the continuity and differentiability of the function under consideration. (Received May 4, 1936.)

\section{Dr. Mary E. Haller: Self-projective rational octavics.}

This paper classifies and discusses self-projective rational octavics, that is, those rational curves of the eighth order invariant under finite collineation groups. By a careful study of curves of orders seven and eight admitting cyclic groups of maximum order, interesting formulas connecting the Plücker constants are observed. These depend upon the order of the multiple point whose parameters are the fixed points of the binary involution. These formulas are generalized and proved to hold true in the general case. The following tables are included: (a) the parametric point equations (in canonical form) of the octavics invariant under cyclic and dihedral groups of orders $m$ and $2 m$, respectively $(m<8)$; (b) the parametric point equations of the curves admitting cyclic and dihedral groups of orders 8 and 16, respectively, and the one octavic invariant under the octahedral group. The parametric line equations, flex forms, and ternary point equations are also given for these curves. (Received May 5, 1936.)

250. Dr. D. H. Lehmer: An algorithm associated with the cotangent function.

The approximation of a real number by a sequence of rationals may in general be accomplished by the continued iteration of a rational function $R(x, y)$ of two variables. Thus the function $R(x, y)=x+y^{-1}$ gives rise to the regular continued fraction algorithm. The algorithm associated with $R(x, y)$ $=x(1-y)$ has been studied by $\mathrm{T}$. A. Pierce. This paper deals with the case $R(x, y)=(x y+1) /(x-y)$. The resulting algorithm yields the development of a number as the cotangent of a sum of arccotangents of integers. This development, which converges with extreme rapidity, has many features in common with continued fractions. (Received May 5, 1936.)

251. Dr. D. H. Lehmer: On functions possessing polynomial addition theorems.

This paper gives the proofs of the following theorems: (1) Let $f(x)$ be a differentiable function possessing a polynomial addition theorem so that 
$f(x+y)=P(f(x), f(y))$, where $P(u, v)$ is a polynomial. Then either $f(x)=a x+b$ or $f(x)=a e^{\lambda x}+b$. (2) Let $f(x)$ be a differentiable function for which $f(x y)$ $=P(f(x), f(y))$. Then either $f(x)=a \log x+b$ or else $f(x)=a x^{\lambda}+b$. These are instances of the following general theorem. (3) Let $\phi(x, y)$ be an arbitrary symmetric function for which $\phi(x, \phi(y, z))$ is also a symmetric function, and let $f(x)$ be a single valued function for which $f(\phi(x, y))$ is expressible as a polynomial in $f(x)$ and $f(y)$. Then $f(x)=a F(x)+b$, where $F(x)$ is any solution of either of the two functional equations: $F(\phi(x, y))=F(x)+F(y)$ or $F(\phi(x, y))$ $=F(x) F(y)$. (Received May 5, 1936.)

\section{Dr. I. M. Hostetter: An extension of Gibbs' vector analysis} to $n$-space.

The method of extension herein employed differs from that used by Weatherburn (See Messenger of Mathematics, vols. 49 and 50 (1919-21)) and others in the development of Gibbs' analysis for 4-space in the definition of the cross product. The product $A \mathbf{X} B$ of the vectors $A$ and $B$ is defined as an anti-self-conjugate dyadic of order $n-2$ which can be reduced to the form $e \alpha \epsilon_{j_{3} j_{4} \ldots j_{n}} i_{j_{3}} i_{j_{4}} \cdots i_{j_{n}},\left(j_{k}=i, \cdots, n-2\right)$, where $\alpha$ is equal to the magnitudes of the vectors $A$ and $B$ multiplied by the sine of the euclidean angle between them, $\boldsymbol{i}_{j}$ are a set of mutually orthogonal unit vectors normal to $\boldsymbol{A}$ and $\boldsymbol{B}$, and $e$ has the value $(-1)^{(n-2)(n-3) / 2}$. An $n$-fold scalar product, a vector product, reciprocal vectors, and $n-1$ polyadics corresponding to the second and third of a dyadic as defined by Gibbs together with the $n$ principal scalar invariants of a dyadic are defined in terms of this product. There follows a brief discussion of two dyadic operators, $\boldsymbol{\nabla}_{\bar{r}} \boldsymbol{r} \cdot$ and $\boldsymbol{\nabla}_{\bar{t}} t \cdot$, which prove useful in the treatment of the geometry of subspaces. (Received May 6, 1936.)

\section{Dr. I. M. Hostetter: A vector treatment of the first neces- sary condition in the problem of Lagrange with finite equations.}

The problem is to determine among the arcs satisfying the $p$ conditions $\phi_{\alpha}(\boldsymbol{r})=c_{\alpha},(\alpha=1, \cdots, p<n-1)$, and passing through two given points, that which minimizes the integral $J=\int_{\tau_{0}}^{\tau_{1}} f(\boldsymbol{r}, \boldsymbol{t})(d s / d \tau) d \tau$. Variations of the form $\boldsymbol{\epsilon}\left(\nabla_{\mathbf{r}} \boldsymbol{r} \cdot \boldsymbol{m}+\eta_{\alpha} \boldsymbol{\nabla} \phi_{\alpha}\right)$ are selected, where $\boldsymbol{\nabla}_{\boldsymbol{r}} \boldsymbol{r}$ is shown to have the value $I-\nabla \phi_{\alpha} \nabla^{\prime} \phi_{\alpha}, I$ being the idemfactor and $\nabla^{\prime} \phi_{\alpha}$ reciprocal vectors of the system $\nabla \phi_{\alpha}, \partial r / \partial u_{\beta},(\alpha=1, \cdots, p, \beta=p+1, \cdots, n)$. Here $m$ is an arbitrary vector function vanishing for $\tau=\tau_{0}, \tau=\tau_{1}$, and the scalar functions $\eta_{\alpha}$ are determined when $m$ is selected. The curve minimizing $J$ must satisfy the vector differential equation $\boldsymbol{V} \equiv \boldsymbol{\nabla}_{\boldsymbol{r}} \boldsymbol{r} \cdot\left(d \boldsymbol{Q} / d s-f_{\boldsymbol{r}}\right)=0$, where the arguments of $\boldsymbol{Q}$ and $f_{\boldsymbol{r}}$ are the $\boldsymbol{r}$ and $t$ satisfying the equations $\phi_{\alpha}(r)=c_{\alpha}, t \cdot \nabla \phi_{\alpha}=0$, and $Q=f_{t}+f t$. The vector $V$ is normal to the extremal arc and is a generalization of vector tangential curvature. For $p=0$ the equation reduces to $V \equiv d Q / d s-f_{r}=0$, and $V$ is again normal to the extremal arc and is now a generalization of vector first curvature. If $f$ is a function of $r$ alone, then the generalized tangential curvature is a section by the $n$ - $p$ space, determined by the equations $\phi_{\alpha}=c_{\alpha}$, of its generalized first curvature in $n$-space. Conditions are shown for the existence of a solution for $V=0$. (Received May 6, 1936.) 
254. Dr. Dean Montgomery: Pointwise periodic homeomorphisms.

Let $M$ be a connected locally euclidean metric space and let $T$ be a homeomorphism of $M$ into itself. If each point of $M$ is periodic under $T$ it is here shown that the periods of all the points of $M$ must have a finite upper bound and accordingly that $T$ itself is periodic. (Received May 6, 1936.)

255. Dr. Deane Montgomery: Continuity in topological groups.

Let $G$ be a locally complete metric space whose elements form a group. It is often assumed that the group combination function $x y$ is continuous in the two variables simultaneously. It is here shown that continuity in each variable separately implies simultaneous continuity. If $G$ is separable the same hypothesis also implies the continuity of the inverse. (Received May 6, 1936.) 